\title{
Giovanni Colombini : un modèle de Catherine de Sienne occulté par ses hagiographes?
}

Giovanni Colombini: un modello per Caterina da Siena occultato dagli agiografi? Giovanni Colombini: a model for Catherina of Siena overlooked by her hagiographers?

\section{Sonia Porzi}

\section{OpenEdition}

\section{Journals}

Édition électronique

URL : http://journals.openedition.org/cei/1013

DOI : $10.4000 /$ cei. 1013

ISSN : 2260-779X

\section{Éditeur}

UGA Éditions/Université Grenoble Alpes

\section{Édition imprimée}

Date de publication : 31 octobre 2012

Pagination : 37-65

ISBN : 978-2-84310-234-9

ISSN : $1770-9571$

Référence électronique

Sonia Porzi, « Giovanni Colombini : un modèle de Catherine de Sienne occulté par ses

hagiographes ? », Cahiers d'études italiennes [En ligne], 15 | 2012, mis en ligne le 30 avril 2014, consulté le 27 mars 2021. URL : http://journals.openedition.org/cei/1013 ; DOI : https://doi.org/10.4000/cei. 1013 


\title{
GIOVANNI COLOMBINI : UN MODĖLE DE CATHERINE DE SIENNE OCCULTÉ PAR SES HAGIOGRAPHES ?
}

\author{
Sonia Porzi \\ Université Blaise Pascal - Clermont-Ferrand 2
}

Principalement connue comme l'auteur du Dialogue, sainte Catherine de Sienne a aussi laissé près de quatre cents lettres et quelques oraisons ${ }^{1}$. Cette abondante production en langue siennoise fait de Catherine Benincasa l'une des premières, sinon la première femme de la péninsule à produire une œuvre importante en langue vernaculaire. Si la rédaction de son traité semble relativement tardive, son abondante correspondance témoigne en revanche d'une pratique de l'écriture épistolaire à la fois ancienne chez elle et complètement inédite pour une jeune lä̈que issue de la petite bourgeoisie artisane ${ }^{2}$. L'originalité de cette démarche n'est que plus évidente quand on considère les figures susceptibles d'avoir marqué les jeunes années de Catherine, qu'il s'agisse de modèles féminins ou masculins, de personnes directement côtoyées par elle ou de personnages plus lointains, connus d'elle à travers les traditions écrite et orale.

Quels sont donc les modèles de Catherine? C'est là une question qui nécessite, d'un point de vue méthodologique, de faire la distinction entre les modèles ouvertement revendiqués par Catherine d'une part, les modèles "officiels», autorisés, mis en avant par son hagiographe Raymond de Capoue d'autre part, et enfin les modèles soigneusement passés sous

I. Pour les œuvres complètes de sainte Catherine, nous nous référerons à l'édition numérique qui propose pour le Dialogue et les Oraisons, la leçon de Giuliana Cavallini, et pour l'édition des lettres, celle d'Antonio Volpato : Santa Caterina da Siena, Opera omnia, Testi e concordanze, CD-Rom, Provincia Romana dei Frati Predicatori, Centro Riviste, Pistoia, 2002. Pour la désignation des lettres, nous renverrons à leur numérotation dans l'édition de N. Tommaseo (par exemple T272): Caterina da Siena, Le lettere, ridotte a miglior lezione, e in ordine nuovo disposte, a cura di Niccolò Tommaseo, 4 vol., Florence, Barbera I860.

2. Sur la question jadis controversée de l'authenticité des œuvres de Catherine dictées par cette prétendue «illeterata» à de savants secrétaires et disciples, voir Sonia Porzi, «Fondement de la persuasion et quête d'autorité dans les Lettres de Catherine de Sienne", Cahiers d'études italiennes, Filigrana, n 2, 2005, p. 63-88. 
silence, occultés pour des raisons de stratégie hagiographique et décelés tardivement par la critique littéraire ${ }^{3}$. Enfin, dans ce domaine, il importe de distinguer les modèles de comportement des simples modèles littéraires parfois réductibles à des sources proprement dites ${ }^{4}$.

Selon la logique de la sequela christi qui se trouve au cour de la démarche spirituelle de Catherine, le Christ est pour elle le modèle absolu de perfection, celui qu'elle prône pour chacun des destinataires de ses lettres. Mais cette figure éblouissante laisse aussi apparaître, dans ses lettres, d'autres personnages exemplaires'. Il s'agit principalement de figures bibliques comme la Vierge et les apôtres, en particulier Pierre et Paul, mais aussi Marthe et Marie-Madeleine auxquelles s'ajoutent les martyres Agnès et Lucie et, marginalement, quelques Pères de l'Église, comme Grégoire cité en exemple aux papes de l'époque. Plus que l'aspect admirable de leurs pouvoirs surnaturels et des miracles qu'ils auraient accomplis, c'est le caractère imitable de ces saints que Catherine met en avant. Tous, ils renvoient à l'Église des origines à la fois rappelée avec nostalgie et brandie avec vigueur comme un projet de société 6 . Il est certain que ces exemples, promus par Catherine pour ses destinataires, ont été aussi ses propres modèles, et plus singulièrement Paul pour son apostolat épistolaire, une modalité de prédication particulièrement adaptée à une femme

3. La Légende majeure [Legenda maior] de Raymond de Capoue est la principale hagiographie de Catherine de Sienne : Raimondo da Capua, S. Catharinae Senensis Vita, "Acta Sanctorum», Aprilis III, Antwerpen, I675, p. 853-959, et pour sa traduction française : Raymond de Capoue, Vie de Sainte Catherine de Sienne, trad. R. P. Hugueny, Paris, Téqui, 1906 (I), 2000. Écrite à l'époque du Grand Schisme d'Occident, cette hagiographie milite en faveur de la canonisation de Catherine à une époque où les détracteurs de cette fervante partisane de Clément V sont encore nombreux. De cette hagiographie dérivent la dilatation [Supplementum] et le résumé [Legenda minor] proposés par Tommaso Caffarini : Tommaso Nacci Caffarini, Libellus de Supplemento Legende Prolixe Virginis beate Catherine de Senis, a cura di Giuliana Cavallini e Imelda Foralosso, Rome, edizioni cateriniane, 1974, et Sanctae Catharinae Senensis legenda minor, a cura di E. Franceschini, Milan, Bocca, 1942, p. X-204. Pour les jeunes années de Catherine, nous disposons aussi de l'œuvre de l'Anonimo Fiorentino, "Questi sono e' miracoli della B. Caterina", dans S. Caterina da Siena, Le Lettere, a cura di Piero Misciattelli, Sienne, Giuntini e Bentivoglio, I92I, vol. VI, p. I83-205. Pour les stratégies hagiographiques de ces différentes œuvres, voir A. Volpato, "Tra Sante profetesse e santi dottori: Caterina da Siena", dans Elisya Schulte Van Kessel (dir.), Women and men in spiritual culture, XIV-XVII centuries, a meetting of South and North, Wetherlands Government Publishing Office, The Hague, I986, p. I49-I6I.

4. Voir Marina Zancan, "Caterina da Siena», Letteratura italiana, Le Opere, Turin, Einaudi, I992, vol. I, p. 593-633, en particulier le chapitre consacré à la question des modèles et des sources de Catherine, p. 615-623.

5. Jésus est présenté par Catherine comme celui dont il faut sans relâche suivre les traces ("seguire le vestigie»), démarche qu'adoptèrent tous les saints devenus par là même imitables à leur tour, comme on peut le lire par exemple dans la lettre Ti83: "Dico che l'anima che è alluminata del vero lume, [...] si dà per la via, cioè per tutte quelle vie e modi che tenne el dolce Gesù, e' santi che 'l seguirono."

6. On notera au passage dans cette dialectique l'écho de certains accents dantesques, en particulier du Paradis où par exemple l'évocation des martyrs et des premiers papes par saint Pierre résonne comme un appel à la réforme morale de l'Église (chant XXVII, v. 40-60) 
du XIV ${ }^{e}$ siècle, dont l'orthodoxie du discours est ainsi rendue aisément contrôlable par la hiérarchie ecclésiastique.

D'après son principal hagiographe, Catherine aurait aussi suivi, enfant, le modèle des Pères du désert, respectivement inspirés par la lecture de la Légende dorée de Jacques de Voragine et la Vie des Pères du désert de saint Jérôme, qui est en effet l'une de ses lectures attestées ${ }^{8}$. Dans la Légende Majeure, Raymond de Capoue souligne l'aspect miraculeux de ces influences sur la jeune femme, comme il le fait aussi pour la figure contemporaine d'Agnès de Montepulciano sur le tombeau de laquelle Catherine viendra plus tard se recueillir' ${ }^{9}$. Raymond de Capoue raconte enfin qu'elle aurait voulu, sur le modèle d'Euphrosine, se déguiser en homme pour entrer chez les Dominicains ${ }^{\mathrm{IO}}$. On peut sans doute voir là l'influence de Tommaso della Fonte, parent éloigné des Benincasa chez qui il grandit avant d'entrer chez les Frères Prêcheurs. De dix ans l'aîné de Catherine, il sera à la fois son confesseur et son premier hagiographe ${ }^{\text {II }}$.

7. La prise de parole des femmes dans l'Église médiévale fait l'objet de contrôles particulièrement attentifs et la prédication telle que l'avait pratiquée Hildegarde de Bingen, est beaucoup plus problématique pour elles à l'époque de Catherine. Raymond de Capoue rappelle en effet les suspicions dont la Siennoise fait l'objet à Sienne, en particulier de la part des Franciscains. L'orthodoxie de la jeune läque est du reste vérifiée à deux reprises, d'abord lors du chapitre général des Dominicains à Florence en 1374, puis lors de son séjour en Avignon en 1376. Sur le profil de Docteur promu par Caffarini dans son hagiographie de Catherine, voir A. Volpato, "Tra Sante profetesse...", art. cit., p. 156.

8. L'Anonimo fiorentino décrit Catherine comme souvent occupée à lire des «libri santi» (où nous pouvons penser qu'elle a pu puiser des modèles), mais sans nous préciser lesquels (Anonimo Fiorentino, "Questi sono e' miracoli...", art. cité, p. 190). Sur la vie des ascètes du désert dont Catherine, enfant, aurait voulu imiter l'exemple en se réfugiant dans une grotte hors de Sienne, voir Raymond de Capoue, Vie..., ouvr. cité, p. 29-30.

9. Raymond de Capoue est l'auteur d'une brève Vita de la bienheureuse Agnès de Montepulciano dont il avait interrogé les témoins directs de sa vie lorsqu'il était confesseur au couvent dominicain de cette ville (Raymond de Capoue, Légende de la bienheureuse Agnès de Montepulciano, Acta Sanctorum, 20 Aprilis, t. 2, I675, p. 79I et suiv.). Catherine ne semble pas, pour sa part, l'avoir personnellement rencontrée de son vivant, mais son pèlerinage sur la tombe d'Agnès, tel que l'évoque Raymond de Capoue, donne lieu à différents miracles. Voir en particulier Raymond de Capoue, Vie..., ouvr. cité, p. 32I-323 où il rapporte le miracle du pied de sainte Agnès qui se soulève pour recevoir le baiser de Catherine.

IO. Les frères dominicains de Camporeggio, voisins du quartier de l'Oca où elle réside, lui fournissent un exemple tout proche dont elle aurait tenté de se rapprocher. Raymond de Capoue évoque en effet l'admiration qu'elle leur porte dès son plus jeune âge, son désir réitéré d'en recevoir l'habit et la promesse que lui en fait, en rêve, saint Dominique lui-même. Sur son rapprochement progressif de l'ordre dominicain, voir différents passages de la Légende majeure : sa dévotion enfantine pour les frères prêcheurs, lorsqu'elle baise le sol après leur passage et sur son projet de se travestir en homme pour être admise dans leur ordre (ibid., p. 35), son souhait d'en recevoir l'habit (p. 5I), la promesse que lui en fait en songe saint Dominique (p. 52) et la demande explicite qu'elle en fait à ses parents (p. 67).

II. Né vers 1337, Tommaso della Fonte est un parent par alliance des Benincasa, puisque Nicolosa, sœur de Catherine, épouse un Palmiero di Nese Della Fonte. Selon le livre des morts de Camporeggio, il meurt le 22 août I390. La première confession qu'il reçoit de Catherine remonterait à la mort de sa sœur Bonaventure en 1362, avant son entrée dans chez les Mantellate. Le recueil des Miraculi (ou Quaderni) rédigé par Della Fonte et aujourd'hui perdu, est ensuite utilisé dans le Supplementum de Caffarini qui en reste l'unique témoignage (Robert Fawtier et Louis Canet, La double expérience de Catherine Benincasa, Paris, Gallimard, I948, p. 69-72 et A. Volpato, "Tra Sante profetesse... », art. cité, p. I49-I50). Confesseur apparemment très apprécié 
Raymond de Capoue ne le présente cependant pas comme un modèle de Catherine, préférant inscrire la candidate à la canonisation dans le sillage de saints reconnus pour leurs pouvoirs surnaturels, des saints plus «admirables» qu' "imitables», pour reprendre la distinction proposée par André Vauchez ${ }^{12}$.

Outre ces figures de référence, directement attestées par les œuvres de Catherine elle-même ou par ses hagiographes, la critique historique et littéraire synthétisée par Marina Zancan, fait émerger toute une série de saintes ou de bienheureuses dont il est cependant difficile d'affirmer avec certitude qu' elles ont pu servir de modèle à Catherine : Claire d'Assise (II93-I253), Marguerite de Cortone (I247-I297), Vanna d'Orvieto (I264I306), Claire de Montefalco (I268-I308), Marguerite de Città di Castello, ou Angèle de Foligno (morte en 1309) ${ }^{\text {r3 }}$. Seules deux d'entre elles ont laissé des écrits, sainte Claire, auteur de cinq lettres, et Angèle dont Marina Zancan considère qu'elle est l'un des modèles les plus importants de Catherine, dans la prise de conscience de la force propre au support écrit. Catherine ne cite jamais le Liber, transcrit en latin par le confesseur d'Angèle, mais on retrouve en effet dans ses lettres certaines de ses positions, en particulier le rapport d'autorité que cette femme illettrée entretient avec les clercs ou encore la tension entre le caractère ineffable de ses visions et l'injonction de diffusion inhérente à ses révélations ${ }^{14}$.

des femmes, Tommaso della Fonte apparaît comme l'un des premiers directeurs de conscience de Catherine dont il perçoit très tôt les dons spirituels, notant bientôt au jour le jour dans des cahiers les paroles et miracles de sa pénitente, à l'instar de frère Rinaldo, le confesseur franciscain d'Angèle de Foligno. Cette initiative suffirait d'ailleurs à tempérer le sévère jugement que porte sur lui Raymond de Capoue selon qui il manque de finesse. Fawtier lui-même a insisté sur les rapports de respect réciproques que Catherine entretiendra avec frère Tommaso tout au long de sa vie : non seulement nous conservons une série de lettres que Catherine lui a adressées, mais ce religieux serait mentionné dans sa lettre-testament du I5 février 1380 . Les lettres que Catherine lui adresse (T283, T4I, T139, T98 et T25) témoignent en effet d'une affection réciproque et durable entre eux.

I2. Dans la typologie qu'il dresse de la sainteté médiévale, A. Vauchez distingue les saints du haut Moyen Âge, admirables pour leurs capacités surnaturelles, et les saints imitables, promus, à partir du XII ${ }^{\mathrm{e}}$ siècle, dans les hagiographies comme des modèles destinés à entraîner la réforme du clergé et, à la suite, de la société tout entière (A. Vauchez, Saints, prophètes et visionnaires, le pouvoir surnaturel au Moyen Âge, Paris, Albin Michel, 1999, p. 56-66).

I3. Voir M. Zancan, "Caterina...", art. cité, p. 62I-623. Il est vrai que les Vies de ces femmes circulaient alors, en version latine, parfois même dans une traduction en langue vulgaire, et il est intéressant de noter que Raymond de Capoue et Caffarini sont particulièrement impliqués dans la diffusion du culte de certaines de ces femmes, le premier étant l'auteur d'une Vie d'Agnès, le second le vulgarisateur des Legendae de Vanna d'Orvieto et de Marguerite de Città di Castello. La Vita de Marguerite de Cortone est écrite au début du XIV $^{\mathrm{e}}$ siècle par Giunta Bevegnati. Pour ce qui est des vulgarisations de ces vies de Bienheureuses, la Legenda della beata Vanna, écrite en 1323 par le Dominicain Giacomo Scalza sera traduite en I40o par Caffarini, ainsi que la Legenda della beata Margherita da Città di Castello, écrite autour de 1348.

I4. Ce recueil de révélations a été élaboré entre I292 et I296 par le franciscain frère Arnaud qui organise la traduction latine de notes prises à partir des descriptions de sa pénitente. En vérité, il serait plus exact de parler au sujet du Liber d'une sorte de polyphonie. Ainsi, dans les quelques pages qu'il consacre à Angèle de Foligno, Alain Boureau montre-t-il bien comment différentes voix, elles-mêmes multiples, se superposent dans le Liber: 
Cette caractéristique prophétique qui se rapprocherait aussi de la fiction dantesque, ne s'associe cependant pas chez Angèle à la largeur de vue de Dante ou de Catherine sur les grandes questions qui animent la vie politique de l'Église. Pour trouver un souffle d'une ampleur comparable, il faut se tourner vers de grandes prophétesses comme Hildegarde de Bingen ou Brigitte de Suède dont il est, là aussi, difficile de savoir si Catherine connaissait leurs œuvres.

Il est en revanche un personnage de son entourage proche, dont Catherine n'a pu ignorer ni l'existence ni l'intense activité épistolaire en langue vernaculaire, un personnage cité comme source littéraire de Catherine tant par Marina Zancan que par Eugenio Dupré Theseider, et dont il est légitime de se demander quelle influence il a pu exercer sur Catherine: c'est le fondateur de l'ordre des Gésuates, Giovanni Colombini ${ }^{15}$. Figure centrale de la vie spirituelle siennoise des années I360 et fervent défenseur du pape Urbain $\mathrm{V}$ dans cette ville de tradition gibeline,

"Angèle à la première personne, Angèle à la troisième personne, Angèle juge de ses propos, Arnaud le scribe, Arnaud le confesseur, Arnaud l'écrivain [...], Arnaud vérifiant son texte auprès d'Angèle", mais aussi celle des autres clercs qui relisent le livre, et enfin celle de Dieu lui-même, qui appose son sceau (sigillabo) et signe (firmabo) le livre (Alain Boureau, Satan hérétique, histoire de la démonologie (I280-I330), Paris, Odile Jacob, 2004, p. 24I-257, et en particulier p. 250-253). Dans ce recueil, Angèle évoque à la première personne son parcours spirituel, jalonné de visions du Christ, et il n'est pas rare de l'entendre déplorer, à la manière de Dante et de Catherine elle-même, l'inefficacité du langage à exprimer cette expérience ineffable : «Oh! ne me faites plus parler. Je ne parle pas, je blasphème; et si j'ouvre la bouche, au lieu de manifester Dieu, je vais le trahir» (Angèle de Foligno, Le livre des visons et des révélations, trad. Ernest Hello, Paris, Seuil, 199I, p. I5I); «souvent, recueillie au fond de moi, j'ai connu avec certitude que plus on a le sentiment de Dieu, moins on peut parler de lui. Plus on a le sentiment de l'infini et de l'indicible, plus on manque de paroles; car auprès de ce qu'on veut rendre, les mots font pitié» (ibid., p. I44); "Dieu est trop au-dessus de l'intelligence et de toute chose; il est trop au-dessus du domaine des paroles, des pensées et des calculs, pour que la bouche essaie d'expliquer parfaitement les mystères de sa bonté. Ce n'est pas que l'âme ait quitté le corps ou que le corps soit privé de ses sens, mais c'est que l'âme perçoit sans leur secours" (ibid.). À différents égards, la démarche d'Angèle annonce en effet celle de Catherine. Tout d'abord dans son rapport avec les spécialistes de l'Écriture que sont pour elle les prêtres et les prédicateurs : ceux qui la «lisent» et la «connaissent»: «Et mon âme vit dans une lumière que ce jugement [que votre vie soit donc lumière et miséricorde pour quiconque la regardera; qu’elle soit justice et jugement pour quiconque ne la regardera pas] serait plus terrible pour les prêtres que pour les laïques, parce que le mépris qu'ils font des choses divines est rendu plus effroyable par la connaissance qu'ils ont des Écritures" (ibid., p. 93); "Il m’est arrivé quelquefois, portée par une ardeur qui voulait sauver : il m’est arrivé de dire quelques secrets; on me répondait : "Ma sœur, revenez à la sainte Écriture"; ou "Nous ne vous comprenons pas" (ibid., p. I4I). À cette connaissance, source d'orgueil, s'oppose selon Angèle, "la science qui n'enfle pas", celle que l'on acquiert en lisant le livre de la vie qu'est le Christ. Pour elle en effet, ceux qui, en suivant l'exemple du Christ, «accomplissent" les Écritures, sont plus grands que ceux qui les «lisent». Non pas qu'Angèle prétende parler de Dieu mieux qu'eux mais, à la différence des prédicateurs par exemple, elle a conscience de son insuffisance. Au-delà de la connaissance acquise à travers la prédication ou la lecture des Écritures, c'est dans l'expérience personnelle que l'on trouve véritablement Dieu, comme elle l'explique dans sa parabole du fils savant. C'est dans cette expérience, plus affective que spéculative, qu'Angèle fonde sa propre autorité, dépassant les obstacles liés à son statut d'illettrée et de femme.

I5. Dans l'apparat critique de son édition des lettres de Catherine, E. Dupré Theseider met en évidence une série d'échos entre les proses des deux Siennois: S. Caterina da Siena, Epistolario di Santa Caterina da Siena a cura di E. Dupré Theseider, vol. I, Rome, 1940, (Fonti per la Storia d'Italia, LXXXII). 
Colombini a pu être pour Catherine un modèle de dévotion pontificale et un exemple vivant de franciscanisme. Pour cerner la personnalité généralement peu connue de Giovanni Colombini et mesurer l'influence qu'il a pu exercer sur le comportement de Catherine, il convient tout d'abord de revenir sur le contexte politique local qui voit fleurir ces deux personnages.

\section{Le gouvernement des Douze à l'épreuve du jongleur de Dieu}

Il est important de garder présente à l'esprit la tradition à l'origine gibeline de Sienne, alliée traditionnelle de l'empereur contre le pape et les guelfes florentins pendant la seconde moitié du Duecento ${ }^{16}$. Instigatrice de la rébellion gibeline en Toscane, Sienne est successivement frappée d'excommunication par les papes Alexandre IV puis Urbain IV ${ }^{17}$. C'est pour échapper aux conséquences économiques désastreuses de cette excommunication que certaines familles de marchands se retirent du gouvernement de Sienne pour fonder en I262 le parti guelfe qui finit par conquérir le pouvoir à Sienne en 1287 , inaugurant ainsi une longue période de prospérité dans laquelle s'inscrit la fresque du «Buon Governo» de Lorenzetti ${ }^{18}$.

Le gouvernement des Neuf (Noveschi) qui reste à la tête de Sienne pendant près de soixante-dix ans, est composé de familles de riches marchands et de banquiers relevant des arts majeurs (mezzagente) ${ }^{19}$. Il se maintient ainsi pendant la première moitié du Trecento, réprimant fortement toute forme de rébellion ${ }^{20}$. Mais cette rigidité croissante alimente le mécontentement du peuple: en I355, à la faveur d'une visite de l'empereur Charles IV à Sienne, le gouvernement des Neuf est renversé par un

I6. Ou «mezzana gente». Pour l'histoire de Sienne, voir J. Hook, Siena: una città e la sua storia, Sienne, La Nuova Immagine, 1988; R. Barzanti, G. Catoni, M. De Gregorio (dir.), Storia di Siena, I-III, Sienne, Alsaba, 1995-1997, et Giuliano Catoni, Breve storia di Siena, Pise, Pacini, 1999.

17. Les Siennois remportent, avec l'empereur Manfred et les nobles bannis de Florence, la victoire de Montaperti en 1260 contre le pape et ses alliés guelfes au pouvoir à Florence. Dans le cadre de cette révolte, Sienne est frappée d'interdit, ce qui dispense les débiteurs des prêteurs siennois de rembourser leurs dettes, plongeant ainsi la ville dans une crise économique. Cet épisode a pu laisser, chez les Siennois, une crainte justifiée de l'interdit, tant sur le plan spirituel que sur le plan économique (ibid., p. I7).

18. Cette fresque, invitant à travailler en ayant confiance dans l'autorité, ornait la salle de la paix du Palais communal de Sienne.

19. On trouve aussi "mezzana gente». Les nobles, ainsi que les juges, les notaires et les médecins étaient exclus de ce gouvernement des Neuf. Pour une analyse des relations entre Nove, popolo et nobles, voir William M. Bowsky, Un Comune italiano nel Medioevo: Siena sotto il regime dei Nove I287-I355, Bologne, Il Mulino, 1986.

20. Parmi ces rébellions, on citera la grève des notaires en $\mathrm{I} 3 \mathrm{I} 8$, la révolte des bouchers, et divers soulèvements fomentés par les nobles, comme les Tolomei ou les Aldobrandeschi (G. Catoni, Breve storia..., ouvr. cité, p. 20). 
soulèvement mené par Giovanni d'Agnolino Salimbeni, avec le soutien des autres nobles, du peuple et le l'Empereur. Excepté une brève parenthèse de coalition avec les Noveschi en I368 (gouvernement des Consuls), l'union des nobles et du popolo parvo reste alors au pouvoir jusqu'en 1385 , d'abord sous le nom de gouvernement des Douze de 1355 à I368, puis, de I368 à I385, sous le nom de gouvernement des Réformateurs (Governo dei Riformatori) ${ }^{21}$.

C'est donc sous le gouvernement des Neuf déjà en phase de déclin que se déroulent les premières années de la vie de Catherine et l'on peut se demander si, après plus d'un demi-siècle de stabilité politique et de prospérité, cette ambiance de crise a pu favoriser chez Catherine une sorte d'identification à certains prophètes de l'Ancien Testament, confrontés à un sentiment d'inexorable déclinn ${ }^{22}$. Mais il convient cependant de rester très prudent car, si le contexte politique siennois des années 1340 est peutêtre à l'origine d'une certaine forme de nostalgie chez Catherine, c'est la nostalgie d'une période florissante au sens large, sans guerres ni épidémies. Catherine a peut-être aussi assimilé l'idée que la prospérité de Sienne est liée à la qualité des rapports qu'elle entretient avec le pape, mais il est difficile d'imaginer chez elle la nostalgie du gouvernement des Neuf en particulier, dans la mesure où elle est issue d'une famille plutôt proche du parti opposé.

Catherine grandit donc dans un contexte général qui ne favorise sans doute pas le soutien inconditionnel qu'elle vouera toute sa vie aux papes, et elle ne semble pas non plus avoir été élevée dans une famille de sensibilité guelfe. Les vers que composait son grand-père matelassier ne semblent pas avoir été d'inspiration religieuse, et parmi ses vingt-quatre frères et

2I. Le gouvernement des Douze, institué en 1355 , est en principe majoritairement populaire, puisqu'il se compose de neuf membres issus du peuple (trois par quartier) contre trois de la noblesse. Mais ce sont en vérité les nobles qui détiennent le pouvoir, le parti des Salimbeni ("Caneschi») rivalisant par ailleurs avec celui des Tolomei ("Grasselli») pour instituer une seigneurie à Sienne. Si le gouvernement des Douze doit faire face aux compagnies de mercenaires qui rançonnent régulièrement la ville, il est aussi confronté à un problème de gestion de l'argent public et de détournement, qu'il tente d'endiguer en renforçant le contrôle financier et en adoptant différentes réformes électorales. Mais en 1368, la mort accidentelle de Giovannino Salimbeni met fin à cet équilibre précaire et, après la parenthèse du gouvernement des Consuls, les nobles Salimbeni reprennent le pouvoir en s'appuyant sur le vicaire impérial Malatesta da Rimini, représentant du popolo minuto : c’est le gouvernement des Riformatori, ainsi nommé car il est chargé de réformer les institutions. La révolte des ouvriers de la corporation des lainiers, menée en I37I par Barbicone, est écrasée dans le sang et les tensions s'exaspèrent entre la volonté d'instituer une "casata grande del popolo» et les dangers d'une autocratie. Un cinquième "Monte» est alors créé, le "monte del popolo" qui, en s'alliant avec les trois premiers («noveschi», "somiglianti» du gouvernement des Douze et "gentiluomini», les nobles) renverse le "monte dei riformatori" et son gouvernement en 1385 ( $i$ bid., p. 22-3I).

22. Sur les prophètes de la chute dans l'Ancien Testament, voir André Néher, Prophètes et prophéties, Paris, Payot, 1972 (I), 2004, p. 226-23I. 
sœurs, aucun n'est entré dans les ordres ${ }^{23}$. Son père, Jacopo di Benincasa, est teinturier dans le quartier de Fontebranda de la Contrada dell'Oca : il relève donc des arts mineurs et il est inscrit, en tant que tel, au popolo parvo, ainsi que ses fils, ce qui ne signifie cependant pas qu'ils soient pauvres $^{24}$. Ce rattachement des Benincasa au popolo parvo favoriserait en revanche leur ralliement au gouvernement des Douze; cette alliance entre le peuple et la noblesse explique peut-être les relations que Catherine, simple fille de teinturier, entretient toute sa vie avec les nobles dames de Sienne (Tolomei, Malavolti, Salimbeni) qui, à leur tour, témoignent plus largement de l'ouverture spirituelle de la noblesse à la prédication de Catherine ${ }^{25}$.

Si la vocation spirituelle de Catherine et son engagement aux côtés de la papauté prend de quelque manière racine dans ses origines, ce n'est donc apparemment ni dans le contexte politique de sa cité ni dans sa famille. Outre l'influence probable de son premier confesseur, le dominicain Tommaso della Fonte, dont le témoignage direct ne nous est cependant pas parvenu, ce qui est susceptible d'avoir orienté la sensibilité de Catherine, c'est le climat de crise lié à la grande peste de 1347, année vraisemblable de sa naissance ${ }^{26}$.

23. On peut se demander pourquoi, comme le fait Fawtier (R. Fawtier et L. Canet, La double..., ouvr. cité, p. 58. Sur son grand-père, voir ibid., p. 48.

24. Voir ibid., p. 48 et E. Dupré Theseider, "Santa Caterina da Siena", Dizionario biografico degli Italiani, vol. XXII, Rome, 1979, p. 361. Les Benincasa semblent en effet relativement aisés puisqu'en 1342, certains des frères aînés de Catherine s'installent à Florence où ils exercent pendant une trentaine d'années (R. Fawtier et L. Canet, La double..., ouvr. cité, p. 5I).

25. La proximité des Benincasa avec le gouvernement des Douze semble confirmée par un épisode de la vie de Catherine qui sauve la vie de deux de ses frères pourchassés lors de la révolte de I368: à la mort de Giovannino Salimbeni, les Douze sont renversés et les Noveschi reprennent partiellement le pouvoir, durant la brève parenthèse du gouvernement de coalition entre nobles et riches marchands (ibid., p. 65-66). Si Catherine a pu être qualifiée de "sainte de l'aristocratie», c'est aussi dans ce sens qu'il conviendrait d'entendre cette proximité. Voir P. Pajardi, Caterina, la santa della politica, Milan, 1993.

26. Les sources hagiographiques sont unanimes pour indiquer que Catherine serait née en I347 (la Légende majeure et ses remaniements ultérieurs par Caffarini, mais aussi les Miracoli de l'Anonimo fiorentino). La date de sa mort en 1380 étant attestée, elle se serait donc éteinte à l'âge de trente-trois ans, ce qui fait suspecter à Fawtier un remaniement de la chronologie catherinienne par ses hagiographes, dans le souci de conformer Catherine, non seulement au Christ, mais aussi à sainte Marie-Madeleine, figure particulièrement chère aux Dominicains et à Catherine en particulier, laquelle aurait mené trente-trois ans de pénitence dans le désert (R. Fawtier, Sainte Catherine de Sienne, Essai de critique des sources, vol. I : les sources hagiographiques, Paris, De Boccard, I922, p. I36-I50; Pierre Mandonnet, "Sainte Catherine de Sienne et la critique moderne», L'année dominicaine, janvier-février 1923; Édouard Jordan, "La date de naissance de Catherine de Sienne", Analecta Bollandiana, XL, I922, p. 365-4II ; R. Fawtier et L. Canet, La double..., ouvr. cité, p. 48). Fawtier propose donc de ramener la date de naissance de Catherine à I337, ce qui permettrait, à son avis, de mieux expliquer certains épisodes de sa vie, liés à la nomination à Sienne de Pietro Adimari, son premier confesseur, à la date de son entrée dans le Tiers Ordre, à l'âge de sa mère et de ses frères, à son influence sur la conversion de Giovanni di Simone alors qu'elle n'aurait eu que quinze ans, à la réputation de sainteté qu'elle aurait eu dès l'âge de vingtcinq ans, alors qu'elle prêchait déjà la croisade. La démonstration de Fawtier, dont il serait trop long de rappeler 
Le XIV ${ }^{e}$ siècle a pu être considéré globalement comme un temps de crises politique, économique et spirituelle, et l'épidémie de peste qui ravage l'Occident par vagues successives de I347 à I44I ne fait qu'accentuer ce climat de catastrophes en chaîne ${ }^{27}$. Sienne n'échappe pas à la peste qui fait périr plus de la moitié de sa population entre $\mathrm{I} 347$ et I348, provoquant une grave crise économique qui contribue à déstabiliser le gouvernement des Neuf ${ }^{28}$. La peste continue à frapper la ville sous le gouvernement des Douze et l'une de ces vagues d'épidémie est interprétée comme une punition divine infligée à la cité à cause du bannissement, en 1363, de Giovanni Colombini, tenu par certains pour un authentique homme de Dieu. Cette interprétation est à l'époque suffisamment répandue à Sienne pour inciter le gouvernement à revenir sur sa sentence et envoyer à Colombini des ambassadeurs l'invitant à regagner sa ville.

Cet épisode, survenu alors que Catherine avait environ seize ans, témoigne d'une tendance alors généralisée, à interpréter la peste ou d'autres maux comme des fléaux de Dieu. Cette conception providentialiste de l'histoire se retrouve dans la lecture que Catherine donne des problèmes de son époque sub specie aeternitatis. Cette grille de lecture, assez simple dans ses premières lettres, ira en s'affinant au fil des ans, Catherine ne se contentant plus de voir la main de Dieu dans les événements, mais cherchant à en expliquer les causes profondes et les finalités lointaines, en les replaçant dans la logique d'ensemble de l'histoire du Salut, dans une perspective eschatologique. Par-delà la conception providentialiste de l'histoire qu'il

ici le détail, repose principalement sur une liste de Mantellate, datée de 1352 et où Catherine figure déjà, ce qui impliquerait, si elle est effectivement née en 1347 , une entrée dans le Tiers-Ordre à l'âge de cinq ans, chose impossible. Contre Fawtier, Jordan, Mandonnet et Taurisano s'accordent pour maintenir une date de naissance en 1347 , s'appuyant en particulier sur l'hypothèse que la liste, écrite par plusieurs mains, a pu servir durant plusieurs années et que Catherine, dont le nom («Katerina Jacobi Benincase») apparaît en cinquante-septième position sur cent, a pu y être inscrite après 1352 (Innocenzo Taurisano, "La critica sulle fonti cateriniane", Studi Cateriniani, ${ }^{\circ}$ I, I923, p. 25; I. Taurisano, "I maestri e i modelli, la vera Caterina da Siena e l'opera del Fawtier", Vita Cristiana, 1949, anno I8, fasc. 3, p. 226). Si Fawtier ne semble pas voir été convaincu par la réfutation de Mandonnet, la date de 1347 semble désormais communément admise par la critique (E. Dupré Theseider, "Santa...", art. cité, p. 36I). À la suite du pape Pie II lui-même, dans sa bulle relative à la canonisation de Catherine, nous jugeons nécessaire d'adjoindre un prudent «circiter» à cette date de I347 («Bolla della canonizzazione di Santa Caterina da Siena, pubblicata da pio II, data in Roma, a San Pietro, il 29 giugno I46I anno terzo del nostro pontificato", Alfredo Scarciglia (dir.), Santa Caterina nei documenti papali, Quaderni Cateriniani, 105, 2002, p. 9).

27. La conséquence démographique de cette épidémie est le phénomène des classes creuses, entraînant la perte de main-d'œuvre et l'affaiblissement de l'économie. Cette catastrophe s'ajoute aux guerres, aux pillages des soldats mercenaires et aux rançons qu'ils réclament, contraignant ainsi les villes à prendre des mesures fiscales exceptionnelles (Yvon-D. Gélinas, "D’Avignon au grand Schisme», Ne dormons plus, il est temps de se lever, Elizabeth J. Lacelle (dir.), Paris-Montréal, Cerf-Fides, I998, p. 19-36, p. 20-29).

28. À titre de comparaison, il suffit de penser au sombre tableau de la peste à Florence, que décrit Boccace dans l'introduction du Décameron (Giovanni Boccaccio, Decameron, a cura di Mario Marti, Milan, Rizzoli, 1974, vol. I, p. 9-17). 
véhicule, l'épisode du bannissement de Colombini témoigne de l'importance de cette figure spirituelle siennoise qui a pu faire partie des modèles de Catherine.

\section{Du nom de Jésus aux « pauvres du Pape ${ }^{29} »$}

Né en 1304, Giovanni Colombini appartient à une ancienne famille noble de Sienne qui, ayant pris part à la République aux côtés du peuple, avait échappé au bannissement de la noblesse au début du XIII ${ }^{\mathrm{e}}$ siècle $^{30}$. Commerçant inscrit à la corporation de la laine, Colombini accroît la richesse dont il a hérité en installant d'importants comptoirs à Pérouse et à Giovanni d'Asso dans le Val d'Orcia, et c'est en qualité de riche marchand qu'il participe lui-même au gouvernement des Neuf, occupant jusqu'à la charge de prieur pendant deux mois ${ }^{31}$.

Il est singulier de noter l'importance des femmes dans son parcours spirituel. C'est sous l'influence de la légende de Marie l'Égyptienne, dont son épouse Biagia lui recommande la lecture, qu'il se convertit en I355. Il s'agit sans doute de la vie proposée par Jacques de Voragine dans la Légende dorée, source privilégiée de modèles «imitables» de sainteté proposés, entre autres, à un public de laïcs parmi lesquels les lectrices ont leur place, comme le montre aussi l'exemple de Catherine elle-même ${ }^{32}$.

29. Sur la vie de Giovanni Colombini, voir en particulier Johannes Joergensen, Colombini, Le feu sacré, Paris, Beauchesne, 1916. Cette biographie reprend principalement celle rédigée par Feo Belcari en I425 (Compendio della vita del beato Giovanni Colombini, testo latino del beato Tavelli da Tossignano e testo volgare di un anonimo del 400 raffrontati con la vita classica del beato compilata da Feo Belcari, a cura di Luigi Albertazzi, Florence, Quaracci, I9IO). Cette œuvre dont la fraîcheur du ton n'est pas sans rappeler les Fioretti, est élaborée par Feo Belcari à partir de deux sources : la vie de Colombini par Cristofano di Gano Guidini mort en I4IO, aujourd'hui perdue, et la Vita et Regula B. Joannis Colombini de Giovanni Tavelli da Tossignano, texte latin que l'on trouve en regard de la vulgarisation de Feo Belcari dans l'édition citée plus haut. Pour les éditions des lettres de Colombini, voir notamment Giovanni Colombini, Le Lettere, Prefazione di Piero Misciattelli, Florence, Libreria editrice Fiorentina, 1923 et Le Lettere del Beato Giovanni Colombini da Siena, a cura di Dino Fantozzi, Lanciano, Carabba, I924.

30. En I223, le noble Ildibrandino Cacciaconti guide le peuple dans soulèvement qui débouchera sur l'institution d'un capitaine du peuple, chargé de défendre le populus (G. Catoni, Breve storia ..., ouvr. cité, p. I5). En I227, certains nobles sont bannis (G. Colombini, Le Lettere, Pref. P. Misciattelli, ouvr. cité, p. 3).

3I. Ibid., p. 3-4. Sur la manière dont il était vêtu avant sa conversion, voir F. Belcari et T. da Tossignano, Compendio..., ouvr. cité, p. 23 (\$ I8).

32. Jacques de Voragine, La légende dorée, trad. Teodor de Wyzewa, Paris Seuil, I900 (I), I998, p. 2I2-2I5. Les légendes d'Euphrosine et de Marie l'Égyptienne, qui semblent avoir marqué respectivement Catherine et Biagia, témoignent en particulier du succès de la Legenda aurea auprès des lectrices issues du milieu artisan, qui y trouvent sans doute l'écho de leurs propres aspirations spirituelles. Le travestissement d'Euphrosine traduit la détermination de Catherine à entrer dans ce qui deviendra, après sa mort, le Tiers Ordre dominicain. Quant à la légende de Marie l'Égyptienne, elle nous ramène à la quête de légitimité des femmes laïques face aux clercs lettrés dans le domaine spirituel. 
La légende de Marie l'Égyptienne évoque l'histoire d'une prostituée du $\mathrm{XIV}^{\mathrm{e}}$ siècle qui raconte les excès de sa vie mondaine, sa conversion, ses tentations et son renoncement définitif au péché, à un ermite imbu de sa science et fier de son mode de vie, auquel Dieu veut donner une leçon en lui montrant l'exemple de cette humble femme qui trouve, dans le seul amour, la force de se purifier et de changer de vie.

À son retour du travail donc, alors que le repas n'est pas prêt et que Colombini s'en plaint à sa femme Biagia, celle-ci lui tend, pour le faire patienter, ce livre qu'il jette d'abord par terre: "Tu non hai altri pensieri che di leggende, a me conviene presto tornare al fondaco ${ }^{33}$." Ce commentaire, apparemment anodin, qui frôle l'accusation de bigoterie, appelle cependant plusieurs remarques. D'une part, il témoigne du contexte domestique dans lequel circule ce type de lecture destiné à un public féminin. D'autre part, cette remarque traduit la vive opposition entre le cadre privé, typiquement féminin, empreint de spiritualité, et le domaine public des affaires, réservé aux hommes, où prédomine alors la mentalité, déjà dénoncé par saint François, des marchands âpres au gain pour lesquels le temps est celui de l'argent compté, prêté, investi, comme le suggère l'impatience de Colombini que sa femme cherche à tempérer ${ }^{34}$.

Ayant finalement lu et médité la légende de Marie l'Égyptienne, Giovanni Colombini réforme sa vie, adoptant des pratiques jusqu'ici inédites chez lui : il fréquente assidûment l'église, prie, jeûne et s'impose la discipline, vit chastement avec sa femme et fait aux pauvres des aumônes toujours plus importantes, avec son ami Mino Vincenti auquel il s'est ouvert de son vœu de devenir pauvre lui-même, sur le modèle plus ou moins explicite de saint François ${ }^{35}$. Si son statut laïc, ses origines de riche marchand et son goût initial pour la vie mondaine de la chevalerie le rapprochent en effet du poverello d'Assise, sa situation familiale d'homme marié et de père de deux enfants, Pietro et Agnese, présente à sa conversion des obstacles supplémentaires auxquels François n'était pas confronté ${ }^{36}$. La mort prématurée de son fils Pietro le dégage d'une partie de ses responsabilités paternelles, sans doute liées à des questions juridiques dans la

33. G. Colombini, Le Lettere... a cura di Dino Fantozzi, ouvr. cité, vol. I, p. 5: «Toi, tu ne penses à rien d'autre qu'à ces légendes; mais moi, il faut que je retourne vite au comptoir.»

34. "La rupture épistémologique décisive des marchands du Moyen Âge consiste en une volonté de contrôler et de mesurer le temps en substituant un temps nouveau, mesurable, c'est-à-dire orienté et prévisible, au temps à la fois éternellement recommencé et perpétuellement imprévisible du monde naturel.» (Jacques Le Goff, Pour un autre Moyen Âge, Paris, Gallimard, 1977, p. 46-47.)

35. F. Belcari et T. da Tossignano, Compendio..., ouvr. cité, p. I8 (\$ 6).

36. Notons cependant, par comparaison à la grande fratrie des Benincasa, que le nombre peu élevé d'enfants de Colombini a pu malgré tout faciliter sa conversion. 
mesure où le renoncement à tous ses biens aurait probablement équivalu, pour son héritier, à une spoliation ${ }^{37}$. C'est sans doute aussi dans ce sens qu'il convient d'interpréter la réticence de sa femme qui, dans un souci d'intendance domestique, lui demande d'être plus mesuré dans ses dons aux pauvres, témoignant par là du conflit qu'il peut y avoir pour un laïc, engagé dans le siècle, entre l'aspiration à l'observance absolue de la pauvreté et une certaine nécessité d'équilibre social. Selon la légende, la disparition miraculeuse d'un pestiféré, soigné et hébergé dans une chambre de la maison des Colombini, a raison des dernières hésitations de Biagia qui, ayant identifié ce malade au Christ lui-même, voit dans cette visite mystérieuse le signe d'une approbation divine face aux choix de son mari auxquels elle consent désormais à se conformer ${ }^{38}$.

Colombini et son ami Vincenti font alors le pas définitif en se séparant de tous leurs biens et en mettant, en I36I, leurs filles, âgées respectivement de treize et de cinq ans, au couvent de bénédictines de Santa Bonda ${ }^{39}$. Là encore, la stricte claustration d'Agnese Colombini permet d'apprécier par comparaison, l'importance de la conquête de Catherine, jeune fille de la même génération qui, à la même époque, combat pour obtenir le statut de tertiaire et rester ainsi dans le monde sans se marier. C'est à ce même couvent de Santa Bonda que Colombini fait don d'un tiers de sa fortune, obligeant en contre-partie les sœurs, selon un acte notarié de 1364, à entretenir jusqu'à leur mort, sa femme Biagia et ses servantes. Pour ce qui est du reste de ses biens, ils sont répartis entre l'Hôpital de Sienne et la Compagnie de la Vierge qui s'y rattache ${ }^{40}$.

Ayant appliqué à la lettre la précepte du Christ déjà suivi par saint François ${ }^{41}$, Colombini et Vincenti vivent dès lors vêtus de loques, pieds et tête nus, mendiant leur nourriture, criant le nom de Jésus ("Gesù» d'où ils tiennent le nom de «Gesuati», Gésuates), et essuyant les moqueries qu'ils

37. "[...] della cui morte Giovanni n'ebbe grande consolazione vedendosi per questo rimaso più spedito e più libero a potere dare ogni cosa per Dio» (F. Belcari et T. da Tossignano, Compendio..., ouvr. cité, p. I4). Cette phrase n'est pas sans rappeler le commentaire d'Angèle de Foligno au sujet de la mort de ses proches. Pour Colombini, il est frappant de voir que sa conversion définitive suit en effet de peu la mort de son fils (G. Colombini, Le Lettere, Pref. P. Misciattelli, ouvr. cité, p. 13).

38. Sur la question du consentement de sa femme, peut-être lié à un problème de dot, voir F. Belcari et T. da Tossignano, Compendio..., ouvr. cité, p. I6-18.

39. G. Colombini, Le Lettere, Prefazione di P. Misciattelli, ouvr. cité, p. I3; G. Colombini, Le Lettere..., a cura di D. Fantozzi, ouvr. cit., vol. I, p. 2I.

40. Ibid. La "compagnia della disciplina della Vergine Maria» est une confrérie laïque dite aussi Santa Maria della Scala (Lettere di S. Caterina da Siena, vergine domenicana, con note di Mons. Lodovico Ferretti, Siena, Tip. S. Caterina, I9I8-I930, 5 vol., vol. V, Indice dei personaggi, p. 432). Il s'agit de l'hôpital de Santa Maria della Scala, développé sous le gouvernement des Neuf, qui cherche à institutionnaliser la bienfaisance.

41. "Se tu vuoi essere perfetto, va e vendi ciò che tu hai, e dàllo a' poveri, e seguita me" (F. Belcari et T. da Tossignano, Compendio..., ouvr. cité, p. 2I). 
provoquent en s'humiliant publiquement, comme lorsque Colombini parcourt en guenilles, monté sur un âne, la Piazza del Campo pour aller se ridiculiser au Palais Communal où il a été naguère honoré comme prieur ${ }^{42}$. On retrouve le même procédé de renversement des valeurs dans cet épisode survenu à San Giovanni d'Asso où Colombini, après avoir donné aux pauvres les terres qu'il y possédait, se fait battre par son ami qui l'injurie publiquement :

Ecco costui che vi volea affamare, che vi prestava ogni anno il gran vecchio punto dalle tignuole, eppoi rivoleva il nuovo buono più che comunale e desiderava che valesse un forino lo stajo! dategli forte a questo crudele odiatore dei poveri! 43

Comme l'a déjà fait remarquer Misciattelli, cet épisode de flagellation, derrière une théâtralité un peu grossière, témoigne en vérité d'une ironie très fine contre toute la noblesse que Colombini remet en cause en s'humiliant lui-même. Le gouvernement des Douze (alliance des nobles avec le peuple), ne s'y est d'ailleurs pas trompé, qui le bannit ainsi que son

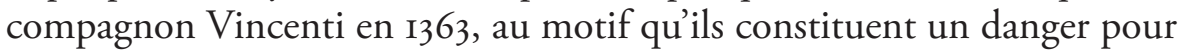
la cité.

La dimension politique de ce danger n'est pas explicitée, mais le gouvernement des Douze a pu craindre que Colombini, cet ancien Novesco, ne fasse, sous couleur de religion, une nouvelle alliance avec le peuple pour renverser les Douze et restaurer les Neuf, tombés précisément l'année de sa conversion, en $\mathbf{3} 355$. Officiellement, le bannissement est motivé par la crainte du dépeuplement de la cité, suite aux nombreuses conversions entraînées par Colombini ${ }^{44}$. Quoi qu'il en soit, ce bannissement perpétuel auquel est condamné Colombini correspond à l'une des formes les plus radicales d'exclusion prévoyant la confiscation des biens, l'interdiction d'hériter et de témoigner, et l'impunité pour celui qui mettrait à mort le banni. Une bougie est allumée à la porte de la ville et la sentence doit être exécutée avant qu'elle ne soit consumée, sous peine de mort.

42. Sur ce rituel public, voir G. Colombini, Le Lettere, Prefazione di P. Misciattelli, ouvr. cité, p. Io. Nous retrouvons peut-être un écho de ce cri dans l'invocation finale des lettres de Catherine "Gesù dolce. Gesù amore».

43. Ibid., p. 8 : "Voilà celui qui voulait vous faire mourir de faim, celui qui vous prêtait chaque l'année le blé ancien, piqué par les charançons, et puis il voulait qu’on lui rende le nouveau, de la meilleure qualité, et il aurait voulu qu'il ne coûte qu'un florin le boisseau! Battez-le durement, ce cruel ennemi des pauvres!"

44. "civitatis depopulationem ac discriminem ex multitudinis conversione ab renuntiantium saeculo", telle est le chef d'accusation prononcé contre Colombini (F. Belcari et T. da Tossignano, Compendio..., ouvr. cité, p. 25). Sur les raisons qui motivent le bannissement de Colombini, voir aussi G. Colombini, Le Lettere, Prefazione di P. Misciattelli, ouvr. cité, p. 9-Io). On devine du reste, en filigrane dans les lettres de Colombini, que les Gésuates font de nombreuses émules (G. Colombini, Le Lettere..., a cura di Dino Fantozzi, ouvr. cité, vol. II, p. I33). 
Parmi les vingt-cinq Gésuates qui suivent alors Colombini dans l'exil, se trouvent des disciples recrutés à Sienne et dans ses alentours (à Montechiello, Montalcino et Asciano) et intégrés au groupe selon un rituel public où ils sont dévêtus devant la représentation de Marie sur le Campo, et rhabillés de loques, tandis qu'on leur ceint la tête de guirlandes d'olivier en chantant des cantiques ${ }^{45}$. Ayant fait vœu d'accepter, à l'instar de saint François, toutes les tribulations dans une joie parfaite, les Gésuates bannis de Sienne prennent alors en chantant la route d'Arezzo puis de Città di Castello et d'Arcidosso, où ils sont bien accueillis et mènent leur prédication itinérante, ponctuée d'épisodes spectaculaires, propres à susciter de nouvelles conversions. Ainsi, à Città di Castello, Colombini ramène-t-il la paix entre familles ennemies, comme les Tolomei et les Piccolomini, qui enterrent la haine qu'ils se vouent depuis l'assassinat de Larino Tolomei, pour se réconcilier ${ }^{46}$. Toujours à Città di Castello, les Gésuates parviennent à convaincre les sœurs du monastère de Sacco de réformer leur règle pour mettre leurs biens en communauté, mesure très franciscaine elle aussi.

C'est entre temps qu'intervient à Sienne l'épidémie de peste évoquée plus haut, aussitôt interprétée par les habitants comme une punition infligée par Dieu à cause du bannissement de Colombini. Sous la pression populaire, le ban prononcé à Sienne contre les Gésuates est levé et on leur envoie des ambassadeurs les invitant à regagner leur cité. Mais en vain, car Colombini, tout en les accueillant aimablement, se dit désormais appelé vers d'autres terres ${ }^{47}$. En effet, il poursuit son apostolat à travers toute la Toscane (à Pise, à Lucques, à Pistoie et à Florence), élargissant ainsi le diamètre de son champ d'action ${ }^{48}$.

Le groupe des Gésuates mène alors une vie faite de prière et de flagellation, dans la pauvreté absolue et la mendicité, refusant d'accéder aux ordres par humilité. Ils revendiquent l'exemple des moines vivant au $\mathrm{IV}^{\mathrm{e}}$ siècle en Thébaïde sous la tutelle de Sérapion d'Arsinoé et travaillant aux champs pour subvenir aux besoins des pauvres, personnages dont Colombini a pu avoir connaissance à travers la Vie des Pères $d u$ Désert qui circule alors en

45. Parmi ses disciples siennois, on compte Tommaso di Guelfaccio, Domenico da Montechiello et sa femme Monna Antonia, Fazio di Berto et Barna di Montalcino, chanteur de laudes (voir ibid., vol. I, p. 22). On retrouve dans les lettres de Colombini un écho du rituel auquel sont soumis les nouveaux convertis: "Sappiate carissimi, che a me venne un figlio di Niccolò di Verdusa, giovane di più di vent'anni e tocco molto da Dio con molto fervore [...] Onde che noi andammo al Campo, e dette le venie alla Madonna, andammone alla fonte, et ine el feci iscalzare a cierti giovani, e puoi andammo a la Madonna del campo, e spogliamolo, e vestimolo piu vilmente, et ine cantammo il Boccia e io una lalda, e puoi il menammo in mezzo, e ndammone a duomo. Or pensate che gran parte della città vi si raccolse, e cosi gli faciemmo per amore di Cristo questa mortificazione e questo vitopero. Disse che pati tanta pena che la morte non è più." (Ibid., vol. II, p. 94-95).

46. Voir ibid., vol. I, p. 22.

47. Voir G. Colombini, Le Lettere, Prefazione di P. Misciattelli, ouvr. cité, p. Io.

48. Voir G. Colombini, Le Lettere..., a cura di Dino Fantozzi, ouvr. cité, vol. I, p. 23. 
traduction italienne, forme sous laquelle elle a sans doute également été lue par Catherine.

Nous avons évoqué l'importance des femmes dans la conversion de Colombini et l'intérêt qu'il porte à différents monastères, en particulier celui de Santa Bonda, où résident désormais sa femme et sa fille et que rejoignent nombre de ses nouvelles converties. La première d'entre d'elles est sa cousine Caterina à laquelle s'ajouteront d'autres Siennoises; quand elles n'entrent pas au couvent, elles vivent pauvrement et honnêtement en travaillant de leurs mains, allant pieds nus, vêtues simplement, la tête bien couverte ${ }^{49}$. Mais la figure féminine principale de la vie de Colombini reste la mère supérieure du couvent de Santa Bonda, Paola di Ghino Foresi. D'origine noble, c'est une femme de forte personnalité qui s'est rendue à Avignon, avec une moniale de son couvent, pour obtenir d'Innocent VI la réforme de sa règle ${ }^{50}$.

Si pendant ces années d'apostolat toscan, les Gésuates font de nombreuses recrues, toutes ne parviennent cependant pas à supporter la rigueur de cette nouvelle vie et s'éloignent de la confrérie. Colombini s'en montre très préoccupé, sans doute conscient de l'irréversibilité du choix de ceux qui, pour le rejoindre, ont renoncé à tous leurs biens ${ }^{\text {sI }}$. Une fois coupés de la confrérie gésuate, ces anciens membres sont en effet promis à une marginalisation sociale parfois fatale, comme le montre l'exemple du frère Biliatto qui, quelques temps après s'être séparé de ses compagnons, est pendu à Arezzo ${ }^{52}$. À cette époque, la pauvreté volontaire est facilement

49. Parmi les Siennoises converties par Colombini, on compte Caterina, sa cousine (la fille de Tommaso Colombini qui est le frère de Piero Colombini, le père de Giovanni), Giovanna di Francesco Marescotti, Francesca d'Ambrogio d'Angnolino, Simona di Ristoro di messer Fazio Gallerani (G. Colombini, Le Lettere..., a cura di Dino Fantozzi, ouvr. cité, vol. I, p. 23). Nous savons d'ailleurs que Catherine Colombini, morte en 1388 , est la fondatrice de la branche féminine des Gésuates (Catherine de Sienne, Lettres, trad. d'Étienne Cartier, Paris, Tequi-Poussielgue, I886 (I), I976, vol. 2, p. I293).

50. G. Colombini, Le Lettere, Prefazione di P. Misciattelli, ouvr. cité, p. I2-I3.

5I. Sur la question des frères qui s'éloignent des Gésuates, voir ibid., G. Colombini, Le Lettere, Prefazione di P. Misciattelli, ouvr. cité, p. I5; G. Colombini, Le Lettere..., a cura di Dino Fantozzi, ouvr. cité, vol. I, p. 23-24. On retrouve un écho de cette préoccupation dans les lettres de Colombini : "Ma quello che peggio ci fa si è che de’ povaregli una parte ànno molto provato male. Unde da Cristo so si partiti, rotornando alla miseria del mondo; unde di loro è molto da dolere" (ibid., vol. II, p. I09); "coloro che, partiti dalla grazia del nostro Signore, tornati sono al misaro mondo e all'abominazione de' peccati, con tanta disgrazia e vitopero, che, secondo che noi udiamo, non è chi loro voglia vedere" (ibid., vol. II, p. I36). Colombini semble particulièrement inquiet d'un certain Minuccio, qui s'est éloigné du groupe : "E mirate come riesce chi si parte dalla brigata. Minuccio andò a Monte Oliveto, e partissene senza far motto. Puoi venne a me, e partissi senza farmi motto. Aitilo Iddio, che bisogno gli fá, per la presunzione e per la superbia» (ibid., vol. II, p. 98); "vedete che Minuccio, per volere avere suo parere, come infino da qui è ito. Partissi da' frati senza far lo motto: abbiatene compassione e pregate per lui; Cristo il guardi. Tutto gli avviene per essere uscito dal parere de' suoi frategli e volere fare per molti consigli. Vanni vi dirà il fatto. Fecemi gran dolore.» (G. Colombini, Le Lettere, Prefazione di P. Misciattelli, ouvr. cité, p. I5.)

52. Au sujet de Biliatto, voir ibid., p. I7. Sa mort semble faire écho à un avertissement reçu par les Gésuates : "sappiate che noi avemo avute novelle che ser Biliatto fu impiccato ad Arezzo con quattordici compagni, si che 
suspectée d'hérésie et les Gésuates courent à tout moment le risque d'être assimilés aux Fraticelles, condamnés et combattus par la curie avignonnaise ${ }^{53}$. Cette suspicion, dont l'ombre inquiétante plane dans la correspondance de Colombini, n'est en vérité pas fondée, les Gésuates prônant, à l'instar de saint François, le respect de la hiérarchie ecclésiastique ${ }^{54}$ : comme le Poverello, ils se placent sous la protection de l'évêque, et comme lui, ils cherchent à faire approuver leur mode de vie par le pape 55 .

C'est dans cette intention qu'ils décident d'aller accueillir Urbain V, premier pape avignonnais à tenter de revenir occuper son siège romain, délaissé depuis soixante ans par les pontifes. Pour se rendre à Viterbe où le pape doit arriver, ce groupe de soixante-dix Gésuates traverse les terres de

vedete che lassando Dio, esso il mando colà uve diprima doveva. Qua mori uno dè povaregli che aveva nome Petrino, e santamente è morto con divozione e fervore, era spogliato de' di che si spogliò ser Biliatto; mirate il giudizio di dio com'è fatto; però è molto da temere" (G. Colombini, Le Lettere..., a cura di Dino Fantozzi, ouvr. cité, vol. II, p. I07); "disse il Nero da Castello che noi avevamo a passare un grande fiume et ine si parrebbe chi fosse di Dio, e molti ne passerebbero; pare ch'esso abbia detto vero. Ser Biliatto che fu il primo fu impiccato ad Arezzo; sapete che non è nuovo, che sempre fu così, e tutti e frutti non vanno a bene, e quando e gattivi ne son fuore, gli altri rimangono migliori, e pero rallegratevi» (ibid., vol. II, p. Io6). Notons au passage l'image du pourrissement des fruits et du risque de propagation que l'on retrouvera, beaucoup plus développé, chez Catherine qui l'applique au corps mystique de l'Église.

53. L'usus pauper est en effet été condamné par Jean XXII et Urbain V veut vêtir les Gésuates d'une tunique blanche pour les distinguer des Fraticelles (G. Colombini, Le Lettere, Pref. P. Misciattelli, ouvr. cité, p. I5). Colombini semble prendre a posteriori conscience du risque qu'ils courent en faisant vœu de pauvreté avec ses compagnons et il fait allusion à ces hérétiques qui ont embrassé la pauvreté absolue : «[...] maraviglia grande è come sia chi ci voglia veder, e come non ci ànno presi e morti. Che per cierto tenete che chiunque è diventato povaro abbia presa opinione o resia, é subito ereticano contro la Chiesa, unde noi, non essendo avisati, ci semo messi a grande rischio. [...] E per certo tenete che se la povertà è qui a sospetto, questo non è colpa di coloro che reggono la Chiesa, ma de' povari superbi ed erranti. E quando costoro sapranno alcuno che viva puro e senza malizia o oppinione, dicianvi che non saranno iscacciati, però che noi semo da tagli iscacciati e villaneggiati che, intesa nostra opinione, ci fanno amore e cortesia, e se vivaremo bene credo che avremo anco di molte e grandi grazie." (G. Colombini, Le Lettere..., a cura di D. Fantozzi, ouvr. cit., vol. II, p. II4.)

54. Voir G. Colombini, Le Lettere, Prefazione di P. Misciattelli, ouvr. cité, p. I7-I8. Nous retrouvons dans les lettres de Colombini de nombreux échos de ce respect inconditionnel de la hiérarchie ecclésiastique, depuis les Dominicains jusqu'aux évêques et aux cardinaux, sans compter le pape lui-même : "Carissimi frategli [Frati di San Domenico] in Cristo Jesù, per mezzo della sua carità, avegna che per l'autorità io vi debba tenere e tenga padri spirituagli, ma la tenerezza dell'amore mi vi fa dire frategli et amici carissimi per Jesù Cristo benedetto" (G. Colombini, Le Lettere..., a cura di Dino Fantozzi, ouvr. cité, vol. II, p. 93); "frategli nostri, sollecitatevi in ogni virtù, e di essare veri ubbidienti a ogni criatura, e spezialmente a' prelati e religiosi e rettori della santa Chiesa" (ibid., p. 132). La lourdeur avec laquelle Colombini proclame parfois son respect de la hiérarchie est à replacer dans le contexte de suspicion d'hérésie dans lequel il écrit. On ne trouve d'ailleurs pas chez lui de condamnation des dérives du clergé, comme chez Catherine.

55. C'est sur le conseil de l'évêque de Città di Castello avec lequel ils correspondent, que les Gésuates renoncent à demander des privilèges au pape pour assurer leur protection ("fate che la virtù vi difenda e non le bolle papli», F. Belcari et T. da Tossignano, Compendio..., ouvr. cité, p. 33). Les lettres de Colombini témoignent largement de sa volonté de faire approuver son mode de vie par le pape, auquel ils se montrent en toute transparence : "Francesco parlò al santo Padre, e disse che vuoleva sapere di nostra vita, e questo è a noi molto di piacere; desideriamo d'essare chiari d'ogni nostro vivare, si che per noi non si possa giammai errare» (G. Colombini, Le Lettere..., a cura di Dino Fantozzi, ouvr. cité, vol. II, p. I09); "Noi ci semo mostrati al santo Padre, et egli e ogni persona vede e fatti nostri palesi" (ibid., p. I22). 
l'Église dans lesquelles ils sont bien accueillis, et où ils recrutent dans leurs rangs Bianco da Siena, connu pour ses laudes ${ }^{56}$. Quand Urbain V arrive à Corneto le 4 juin 1367 , ils l'accueillent avec des chants et des vivats, puis le suivent à pied jusqu'à Toscanella où ils sont ensuite convoqués pour une première entrevue avec le souverain pontifes7. Les ayant interrogés sur leurs règles de vie, ce dernier conditionne son approbation à un examen serré de leur orthodoxie mise en doute par de nombreux membres de la Curie $^{58}$. Examinés par le cardinal de Marseille, dominicain, et par l'inquisiteur de Viterbe, Colombini et ses compagnons profèrent leur obéissance à l'Église et au pape qui les approuve, les revêtant de blanc 59 . Sur le conseil de leur protecteur, le cardinal d'Avignon, frère d'Urbain V, ceux qu'on appelle dès lors à Viterbe «les pauvres du pape» se diviseront en familles, s'établissant en différents lieux de Toscane avec le consentement de l'évêque diocésain ${ }^{60}$. Leurs privilèges seront confirmés par Grégoire XI

56. Voir ibid., vol. I, p. 23.

57. Voir F. Belcari et T. da Tossignano, Compendio..., ouvr. cité, p. 29-30, G. Colombini, Le Lettere, Prefazione di P. Misciattelli, ouvr. cité, p. I7-I8. Colombini raconte lui-même l'arrivée du pontife et leurs premiers contacts avec lui : à Corneto, ils ont attendu le pape sur le pont et son arrivée est la plus belle chose jamais vue; leurs têtes ceintes de rameaux d'olivier, ils criaient "louange à Jésus » et "vive le pape » et ils ont ainsi mis un air de fête dans toute l'assemblée. Là se trouvait toute la baronie du monde; ils ont baisé le pied du pape. Arrivé à terre, il chevauchait entouré des Franciscains et des Gésuates. Le pape à qui l'on parle d'eux exprime le désir de les rencontrer, mais cela ne peut se faire dans un premier temps. Ils rencontrent cependant le cardinal d'Avignon frère du pape qui les conseille avec bienveillance. Ils sont beaucoup aimés et les plus remarqués de tous, de sorte que leur renommée s'étend depuis à toute la chrétienté (G. Colombini, Le Lettere..., a cura di Dino Fantozzi, ouvr. cité, vol. II, p. IOI-IO3). Ils ont accompagné le pape depuis son arrivée, puis de Corneto à Toscanella ils l'entourent en courant autour de lui, celui-ci manifeste sa bienveillance à leur égard, comme Francesco lui baise le pied, il retient son cheval, et Francesco retient ses vêtements quand il traverse les rivières. À Toscanella, le pape cherche en vain les Gésuates et le soir quelqu'un vient les quérir pour les conduire devant lui. Appelé devant le pape, Colombini jette son manteau et s'agenouille, le pontife le fait approcher et l'interroge sur leur mode de vie et déclare préférer qu'ils soient mieux vêtus tout en restant déchaussés : il veut leur faire faire des robes (ibid., vol. II, p. IO4-IO5).

58. Colombini raconte cet examen dans ses lettres : le pape a voulu faire examiner les Gésuates par le cardinal de Marseille, un Dominicain; ils ont ainsi été convoqués devant lui, entouré d'un inquisiteur et d'un notaire muni d'une feuille blanche. Ils ont été interrogés contre la volonté du cardinal d'Avignon qui craignait pour eux. Mais l'interrogatoire s'est bien déroulé, le cardinal a été ravi : il n'a rien écrit et est resté à discuter avec eux, les traitant comme ses fils et leur proposant son aide, les invitant à manger et leur proposant le lendemain d'aller à la messe dans la chapelle du pape. Il les a soutenus contre tous leurs détracteurs et tous les maîtres et frères qui les questionnaient sur leur pauvreté (ibid., vol. II, p. II2-II3).

59. Au sujet du nombre de robes, voir F. Belcari et T. da Tossignano, Compendio..., ouvr. cité, p. 29-3I. On peut suivre dans les lettres de Colombini l'évolution de ce projet, le pape ayant d'abord promis de vêtir vingt-cinq Gésuates, puis ayant fait réaliser cinquante robes, et enfin soixante (ibid., vol. II, p. II2-II9, I37). En les vêtant de blanc, le pape veut sans doute les distinguer ostensiblement des Fraticelles, peut-être aussi pour les protéger. Sur la volonté du pape de les vêtir, voir ibid., vol. I, p. 24 et F. Belcari et T. da Tossignano, Compendio..., ouvr. cité, p. 30-3I.

6o. On les a conseillés au sujet de la confession : ceux qui n'ont pas de maison ou d'asile peuvent se confesser auprès de religieux sans licence, en revanche, ceux qui ont un domicile ont besoin de la licence de leur parrain (G. Colombini, Le Lettere..., a cura di Dino Fantozzi, ouvr. cité, vol. II, p. II4-II5). 
et par ses successeurs ${ }^{6}$. Colombini, pour sa part, n'assiste pas à cette structuration des Gésuates, puisqu'il meurt le 31 juillet 1367 , sur le chemin du retour vers le monastère de Santa Bonda où il est enseveli selon ses dernières volontés le 2 août suivant, en présence de Catherine de Sienne ${ }^{62}$.

\section{Un modèle vivant de franciscanisme pour Catherine}

On a souvent évoqué le fait que Catherine aurait assisté aux funérailles de Colombini, mais cela ne prouve pas en soi qu'elle l'ait fréquenté de son vivant. Ce qui n'a en revanche pas été suffisamment souligné, c'est le dense réseau de relations sociales communes aux deux personnages. Tout d'abord, Catherine et Colombini étaient parents par alliance puisque Lisa Colombini, la nièce de Giovanni, était la belle-sœur bien-aimée de Catherine. Par ailleurs, leurs correspondances respectives font apparaittre des connaissances communes comme Matteo Colombini, cousin germain de Giovanni Colombini qui est aussi un correspondant de Catherine (T48), ou encore Moranda, épouse de Francesco di Monte Alcino, destinatrice d'une lettre de Colombini (FI6) et qui est évoquée par Catherine dans la lettre Ti2, sans parler des moniales de Santa Bonda, principales destinataires de Colombini, qui sont aussi des correspondantes de Catherine $\left(\mathrm{TT}_{73}, \mathrm{~T}_{220}\right)^{63}$. À ces connaissances communes, il conviendrait sans doute d'ajouter Bartolomeo Montucci qui fut l'un des premiers confesseurs de Catherine, et qui était probablement parent par alliance d'une sœur de Giovanni Colombini entrée ensuite dans les ordres ${ }^{64}$. Mais le lien le plus fort entre les deux épistoliers concerne leurs plus proches collaborateurs : non seulement Guidini, disciple de Catherine, est le traducteur d'une vie de Colombini (ce qui pourrait, certes, s'expliquer par un goût plus ou moins tardif pour la littérature spirituelle de l'époque), mais, coïncidence plus probante, Tommaso di Guelfaccio et Santi di Teramo,

6I. Voir F. Belcari et T. da Tossignano, Compendio..., ouvr. cité, p. 3I-34.

62. Sur la mort de Colombini et son testament, voir ibid., p. 34-37; G. Colombini, Le Lettere, Prefazione di P. Misciattelli, ouvr. cité, p. 22-23 et G. Colombini, Le Lettere..., a cura di Dino Fantozzi, ouvr. cité, vol. I, p. 25. Sur la présence de Catherine aux funérailles de Colombini, voir S. Caterina da Siena, Le Lettere, a cura di D. Umberto Meattini, premessa di Oscar Luigi Scalfaro, Milano, Edizioni Paoline, 1997, p. IO29.

63. Pour l'allusion à la fille de Moranda dans la lettre Ti2 : «ella volrebbe rinchiudarle per modo che a me non piace tanto". Sur Lisa Colombini, voir R. Fawtier et L. Canet, La double..., ouvr. cité, p. 56. Sur les Gésuates devenus secrétaires de Catherine, voir ibid., p. 74. Au sujet de Bartolomeo Montucci, voir ibid., p. 57.

64. Voir ibid., p. 57. 
anciens Gésuates, sont devenus disciples et secrétaires de Catherine à la mort de Colombini ${ }^{65}$.

On le voit, la présence de Catherine aux funérailles de Colombini en I367 ne semble pas anodine. De même, le fait que l'année de la mort de Colombini corresponde à l'entrée de Catherine sur la scène publique de la vie spirituelle siennoise apparaît comme bien plus qu'une simple coïncidence : il semble s'agir là d'un véritable passage de flambeau ${ }^{66}$. S'il apparaît clairement que Catherine a eu de multiples points de contact avec le fondateur des Gésuates, on ne trouve cependant aucune trace d'échange direct entre ces deux figures religieuses majeures de la Sienne du XIV ${ }^{\mathrm{e}}$ siècle, ni dans leurs hagiographies ni dans leurs correspondances respectives.

On peut s'interroger sur les causes de ce silence. S'agit-il d'une forme de censure des hagiographes et des compilateurs sur un modèle inavouable de Catherine, et si tel est le cas, pour quelle raison? Ce silence témoignet-il de conflits entre Catheriniens et Gésuates de la deuxième génération, comme le laisseraient présumer certains passages polémiques des laudes de Bianco da Siena qui met Catherine en garde contre la tentation du "profetico sermone", l'accusant ainsi de s'abandonner à de sombres prophéties sans doute comparables à celles délivrées naguère par Brigitte de Suède ${ }^{67}$ ? Pour répondre à ces questions, il convient de s'interroger maintenant sur les éventuelles similitudes entre Catherine et Colombini, tout d'abord dans leur démarche générale, puis dans leur style épistolaire en particulier.

Pour Catherine comme pour ces contemporains siennois, Colombini incarne l'esprit franciscain des origines, il est un exemple vivant de franciscanisme, antérieur aux contacts que Catherine pourra entretenir par la suite avec de savants frères mineurs tel William Flete qui représente une autre tendance de l'ordre. L'esprit franciscain dont Catherine aurait subi

65. Au sujet de l'influence probable des secrétaires gésuates et de leurs habitudes de présentation des lettres, relevons que l'on trouve déjà chez Colombini, la formule "altro non diciamo" au beau milieu d'une lettre, comme si l'épistolier ne pouvait se résoudre au silence et poursuivait sa dictée, ainsi que le fera aussi Catherine (G. Colombini, Le Lettere..., a cura di Dino Fantozzi, ouvr. cité, vol. II, p. IIs par exemple).

66. Notons que c'est aussi à l'année $\mathrm{I}_{3} 67$ que remonte la plus ancienne lettre datable $\left(\mathrm{T}_{30} \mathrm{O}\right)$ qui nous soit parvenue de Catherine (S. Caterina da Siena, Epistolario..., a cura di E. Dupré Theseider, ouvr. cité, p. 3).

67. Laude 72, str. I4: "guarda che la tentazion del profetico sermone non ti metta in suo prigione» (Laudi spirituali del Bianco da Siena, povero gesuato del secolo XIV, codice inedito dato in luce da Telesforo Bini, Lucques, G. Giusti, I85I). Cette laude aurait été écrite avant 1390. On retrouve le même type d'accusation contre la prophétesse dans la laude 32 : «lassa andar le fantasie delle vana profezie». Au sujet de Bianco da Siena, voir en particulier Franca Brambilla Ageno, Il Bianco da Siena: notizie e testi inediti, Genova-Roma-Napoli, Società anonima ed. Dante Alighieri, I939. Selon Ageno, Bianco da Siena serait né entre I345 et I350 et mort en I4I2. Dans les laudes 32 et 72, il exhorte Catherine à ne pas devenir trop hautaine à cause de sa réputation de sainteté et à ne pas s'éloigner de l'humilité (ibid., p. XVI-XVII). 
l'influence à travers Colombini n'est ni conventuel ni spirituel, lui-même ne s'étant rapproché d'aucune des deux branches de l'Ordre des Frères Mineurs. Certes, par certains traits, Catherine se démarque très nettement des positions de Colombini. Il serait vain, par exemple, de chercher dans les lettres destinées par Catherine à des monastères, la moindre invitation à se réformer structurellement dans le sens d'une communauté des biens, prônée comme on l'a vu, par Colombini pour le monastère de Sacco. Cette retenue ne semble pas étrangère, chez Catherine, à ses positions très modérée sur la pauvreté. Sur cette question, Colombini se rapprocherait plutôt des positions radicales de certains spirituels qui ne seront pas reprises par Catherine ${ }^{68}$. On ne trouvera pas dans ses lettres l'euphorie du dépouillement total, qui confère aux conversions des Gésuates cette empreinte typiquement franciscaine. De ce point de vue, on appréciera la nouveauté du compromis inauguré par Catherine, certes restée dans le célibat, mais continuant à vivre dans la maison familiale et faisant preuve d'un véritable sens de l'équilibre entre la vie domestique et les dons aux pauvres $^{69}$. Sur un plan plus collectif, Catherine rappelle certes vigoureusement aux papes que le trésor de l'Église doit être destiné aux pauvres et pour elle, ce trésor temporel reste, quoiqu'il en soit, moins précieux que le pouvoir spirituel, véritable trésor légué par le Christ à son Église. Mais jamais Catherine ne stigmatise pour autant la propriété à proprement parler comme un péché : elle ne fait que rappeler un ordre de priorité face aux dérives de son époque. Sur ce point, les positions prudentes de Catherine la mettent à l'abri de l'assimilation aux Fraticelles alors frappés d'hérésie, une assimilation fâcheuse dont certains Gésuates avaient tragiquement fait les frais, comme nous l'avons vu.

Sur d'autres points, en revanche, Catherine s'inscrit très nettement dans le sillage du franciscanisme de Colombini, comme par exemple dans ses rapports avec la hiérarchie ecclésiastique, et en particulier sur la question de l'obéissance, dont le fondateur de Gésuates fait dans son testament une priorité absolue. En vérité, c'est une injonction qui émaille toute sa correspondance à ses disciples : "Frategli nostri, sollecitatevi in ogni virtù, e di essare veri ubbidienti a ogni criatura, e spezialmente a’ prelati e religiosi

68. Si, pour Catherine, l'Église doit revenir à la pauvreté des origines ("lo stato suo povarello», T206), il ne s'agit pour elle que d'un ordre de priorité, une manière d'exprimer que le spirituel doit primer sur le temporel au sein de l'Église. Elle n'engage d'ailleurs aucun de ses correspondants à donner tous ses biens aux pauvres.

69. Se penchant sur le miracle du vin et du pain que Catherine distribue aux pauvres sur les réserves familiales sans que jamais celles-ci ne s'épuisent, Giovanni Getto en propose une lecture non seulement symbolique, en en dégageant le sens eucharistique, mais aussi sociale (G. Getto, Letteratura religiosa del Trecento, Firenze, Sansoni, I967, p. I2I-I22). 
e rettori della santa Chiesa $^{70}$." Cette révérence, observée par Colombini lui-même, concerne non seulement le clergé séculier, mais aussi le clergé régulier, et en particulier les frères Dominicains ${ }^{71}$. C'est dans le cadre de cette proclamation conjuguée d'obéissance et d'amour envers ses correspondants, que s'inscrit la direction spirituelle exercée par Colombini sur les frères prêcheurs, y compris le supérieur du monastère, auxquels il n'hésite pas à rappeler comment ils doivent prêcher : "Pregovi che sempre oriate per gli uditori, e singularmente questo s'appartiene al soperiore, orate quando predicate $^{72}$. " Nous retrouverons ce dosage subtil de soumission et d'autorité chez Catherine quand elle se permet par exemple, dans une lettre relativement ancienne (1372-I373), de conseiller son confesseur dominicain Bartolomeo Dominici sur la manière de prêcher à Asciano pendant le Carême (T208). Plus tard, c'est au pape lui-même, que la jeune tertiaire s'adressera sur ce mode, qu'il s'agisse de Grégoire XI ou d'Urbain VI.

Nous n'avons pas connaissance de lettres que Colombini aurait adressées à Urbain $\mathrm{V}$, et on l'imagine mal se permettant de donner des conseils au pontife. Toutefois, le déplacement des Gésuates à Corneto pour accueillir le Pontife de retour en terre italienne et obtenir de lui l'approbation de leur règle, se situe à mi-chemin entre le voyage de saint François à Rome devant Innocent III et celui de Catherine à Avignon auprès de Grégoire XI qu'elle tentera d'affermir dans sa volonté de regagner son siège romain.

Tout se passe en fait comme si la dynamique que l'on trouve in nuce dans la démarche de Colombini, trouvait sa pleine expression chez Catherine. Que l'on songe à l'apostolat des Gésuates en Toscane : inspiré de la prédication itinérante de François et ses frères en Ombrie, cet apostolat préfigure la mission menée par Catherine dans le val d'Orcia, à son retour d'Avignon. Il en va de même pour leurs ouvres respectives de pacification. Inscrite dans la continuité des réconciliations opérées par la Siennoise dans sa propre ville, la paix conclue entre différents membres de la noble famille des Salimbeni lors de cet apostolat dans le Val d'Orcia, annonce les missions diplomatiques ensuite menées par Catherine à plus grande échelle encore, entre le pape et les Florentins. L'embryon de cette dynamique se trouvait déjà chez Colombini, lui-même artisan de récon-

70. «Lettera a Fazio e Giovanni d'Ambrogio e suoi compagni povari per Cristo », G. Colombini, Le Lettere..., a cura di Dino Fantozzi, ouvr. cité, vol. II, p. I32 : «Chers frères, exercez-vous à toutes les vertus, et à la véritable obéissance à toute créature, en particulier aux prélats, aux religieux et à ceux qui régissent la sainte Église.»

7I. Ibid., vol. II, p. 93 : "Carissimi frategli [di San Domenico] in Cristo Jesù [...] per l'autorità io vi debba tenere e tenga padri spirituagli, ma la tenerezza dell'amore mi vi fa dire frategli et amici carissimi per Jesù Cristo benedetto."

72. Ibid., vol. II, p. 94 : "Je vous demande de toujours prier pour vos auditeurs, chose qu'il appartient tout en particulier au supérieur de faire, priez quand vous prêchez.» 
ciliations à Città di Castello avant de jouer un rôle d'intermédiaire entre le gouvernement des Douze et Urbain V à qui les Siennois n'avaient pas envoyé d'ambassadeurs lors de son arrivée à Corneto, risquant ainsi de compromettre leurs relations avec le pape ${ }^{73}$. Avec cette démarche, quoique encore très limitée et à peine ébauchée, en faveur de la paix sur le plan politique, Colombini dépasse le cadre de la direction individuelle pour s'orienter vers une action collective.

La vision de Colombini semble en effet prendre de l'ampleur à la fin de sa vie, notamment au moment de sa rencontre avec Urbain V. Les destinataires des prières qu'il "commande" à ses amies moniales en témoignent : des prières pour le pape, les cardinaux et tous les gardiens de l'Église: "Pregate Dio per tutti questi che guardano la Chiesa di Dio, però che molta pena e fatica ànno ${ }^{74}$." De même, on voit émerger discrètement dans ses dernières lettres l'espoir d'avoir été élu par le Christ pour «far grandi cose in onor suo e della santissima Chiesa ${ }^{75}$ ". Colombini meurt peu de temps après, sans avoir eu le loisir de développer d'autres actions de cette envergure, mais il y a là, nous semble-t-il, comme l'impulsion initiale d'une dynamique que l'on retrouvera, beaucoup plus aboutie, chez Catherine dont les préoccupations s'élargiront progressivement du cadre strictement communal à la politique de l'Église au sens large, qu'il s'agisse de la question avignonnaise, de la croisade, de la paix dans les États pontificaux ou du Grand Schisme d'Occident.

Si Colombini semble faire revivre dans les années I360 l'idéal franciscain des origines dont les deux branches de l'ordre se sont, aux dires de Dante, désormais autant éloignées l'une que l'autre, c'est aussi grâce à la place privilégiée que le Gésuate accorde aux femmes. Le recueil de lettres que nous conservons de Colombini témoigne en effet d'une pratique épistolaire assez intense, en particulier à partir de son bannissement qui l'éloigne de ses proches et des moniales de Santa Bonda, avec lesquelles il menait une action de soutien aux pauvres. En filigrane dans sa correspondance, on devine en effet que les Gésuates sont spirituellement très liés aux Bénédictines de Santa Bonda, dont l'œuvre est complémentaire

73. "E però io, costretto dalla carità di Dio, e come zelatore e amatore de la vostra pace e buono stato, profersi a missere Francesco voler mandarvi subito uno de' miei compagni povaregli..." (ibid., vol. II, p. I39).

74. Ibid., vol. II, p. I09 : "Priez pour tous ceux qui gardent l'Église de Dieu car ils ont bien du mal et de la peine.» Mais il ne s'agit pas là d'une citation isolée : "ricordianvi che preghiate per il santo Padre e per tutti $i$ pastori della Chiesa di Dio" (ibid., vol. II, p. IO4); "pregate Dio per noi, per lo santo Padre e per gli cardenagli» (ibid., vol. II, p. I30). Nous retrouverons cette invitation à prier pour le pape chez Catherine qui élargit ensuite cette intention de prière à l'Église tout entière.

75. G. Colombini, Le Lettere..., a cura di Dino Fantozzi, ouvr. cité, vol. II, p. I03 : «réaliser de grandes choses en son honneur et en l'honneur de la très sainte Église». 
à la leur : les Gésuates, itinérants, adressent les pauvres qu'ils rencontrent aux moniales qui en prennent soin dans leur monastère ${ }^{76}$. Cette collaboration ne manque d'ailleurs pas de susciter la suspicion (ou l'intérêt, on ne sait) des autorités ecclésiastiques et du pape lui-même, à qui l'on songeait à adresser, avant l'examen auquel ils furent soumis par le cardinal de Marseille, les résultats d'une première enquête restée sans suite ${ }^{77}$.

Si la supérieure du couvent de Santa Bonda, Paola di Ghino Foresi, a pu être considérée comme la sainte Claire de Colombini, elle n'est pour lui ni la compagne ni la sœur que François voyait dans la noble Chiara degli Offreducci, mais une véritable mère, à laquelle il se soumet avec humilité. Le titre de "madre» par lequel il la désigne dans les nombreuses lettres qu'il lui adresse ("carissima madre in Cristo crucifisso ${ }^{78}$ ) est apparemment lié à sa dignité d'abbesse, mais on note déjà sous sa plume quelques occurrences du terme affectif mamma ("alla mia cara e perfetta mamma e da me molto amata $\left.{ }^{79} »\right)$, terme par lequel Catherine, qui n'est pourtant pas abbesse, sera qualifiée par ses disciples et se désignera d'ailleurs aussi elle-même dans ses lettres (T198 : "Benedicete el mio figliuolo frate Simone; diteli che disponga la bocca del desiderio a ricevare el latte, ché la mamma ne li mandarà $\grave{10}^{80}$ ). S'il ne s'agit pas là d'une pratique commune dans le parler siennois du XIV ${ }^{\mathrm{e}}$ siècle, c'est sans doute également de cet usage que dérive l'emploi de babbo par lequel Catherine qualifie, de manière si originale, le Saint-Père lui-même : "O babbo mio, dolce Cristo in terra", écrit-elle par exemple à Grégoire XI dans la lettre TI8 $85^{81}$. On peut s'interroger sur la portée de ces termes qui, tout en s'inscrivant dans la terminologie familiale de la tradition monastique, revivifient ces titres désormais galvaudés en leur réinsufflant leur dimension affective originelle. Saint François avait déjà renouvelé cette terminologie en substituant au lexique parental (pères

76. "Quanto $[i o]$ sia contento della carità che per tutte voi s'è usata verso questi povaregli non vel potrei dire mai», leur écrit par exemple Colombini (G. Colombini, Le Lettere..., a cura di Dino Fantozzi, ouvr. cité, vol. II, p. I2O-I2I) ou encore "per non cresciare costi gente a darvi angoscia non v'avemo mandati più povaregli, anco gli avemo isparti per li paesi dattorno" (ibid., vol. II, p. II8). Malgré cette collaboration en faveur des pauvres, le couvent de Santa Bonda ne peut pas être considéré comme la «branche» féminine des Gésuates qui comptent des femmes dans leurs rangs, comme le prouvent les lettres adressées par Colombini à «Caterina sua suoro e alle sue compagne e suoro povare per Jesù Cristo e a monna Margarita et a Guelfa sua figliuola" (ibid., vol. II, p. I45).

77. Voir G. Colombini, Le Lettere..., a cura di Dino Fantozzi, ouvr. cité, vol. II, p. I23.

78. G. Colombini, Le Lettere..., a cura di Dino Fantozzi, ouvr. cité, vol. II, p. I26 par exemple.

79. Ibid., vol. II, p. I27.

8o. "Bénissez mon fils frère Simon, dites-lui de présenter la bouche du désir pour recevoir du lait, car sa maman lui en donnera. " Notons qu’au sein du petit groupe de disciples catheriniens, on a coutume de désigner la mère de Catherine, Lapa, de "nonna" : "La nonna e Lisa e tutta l'altra povarella famiglia vi confortano in Cristo" (T332).

8I. "Oh mon papa, doux Christ sur terre". 
et mères supérieurs des monastères) celui de la fratrie (frères et sœurs) appliqué indistinctement à tous les niveaux de la hiérarchie, y compris aux ministres de son ordre auxquels il rappelait par ailleurs qu'ils étaient avant tout des serviteurs de leurs frères, à l'instar du Christ venu pour servir et non pour être servi ${ }^{82}$. Avec Colombini et Catherine de Sienne, on retrouve la terminologie parentale traditionnelle qui à travers la connotation de bienveillance qu'ils véhiculent, rappelle la double dimension des responsabilités hiérarchiques qui ne doivent pas se réduire à une forme de domination et où l'amour est le corollaire indispensable de toute forme d'exercice de l'autorité ${ }^{83}$. L'emploi de titres tels que «mamma" chez Colombini ou "babbo" chez Catherine, apparaissent donc à la fois comme une déclaration d'obéissance, à l'abbesse ou au pape, et comme un appel à la bienveillance, sinon à la clémence et à la miséricorde.

En proférant ainsi son humble respect à Paola Foresi qu'il considère comme un exemple de perfection dans le Christ, Colombini inaugure par ailleurs une valorisation particulière de la figure féminine, peut-être liée au développement du culte marial, en particulier dans les laudes, qui a pu influencer la sensibilité de Catherine, expliquant ainsi en partie l'assurance inouïe dont elle fera preuve en maintes circonstances face aux hommes les plus haut placés des hiérarchies politiques et ecclésiastiques. Il n’est pas exclu que cette exaltation de la figure féminine soit liée chez Colombini, ancien admirateur de la littérature chevaleresques, à des schémas de la poésie courtoise spiritualisés dans le sillage de saint François, non seulement chevalier du Christ et saltimbanque de Dieu ("giullare di Dio»), mais aussi époux de Dame Pauvreté. On retrouve d'ailleurs dans les lettres de Colombini l'écho de cette allégresse typiquement franciscaine dont les accents candides rappellent parfois le ton des Fioretti ${ }^{84}$.

82. Saint François d'Assise, Première Règle des Frères Mineurs (I22I), dans Saint François d'Assise, Documents, Ecrits et premières biographies, Théophile Desbonnets et Damien Vorreux (dir.), Paris, Éditions Franciscaines, 1968, ch. 4,2 et 4,6, p. 58. Il s'agit d'un écho de Mt 20, 28 ("sicut Filius hominis non venit ministrari sed ministrare et dare animam suam redemptionem pro multis").

83. Sur la nouveauté que représente la terminologie fraternelle inaugurée par saint François, voir A. Vauchez, François d'Assise, Paris, Fayard, 2009, p. 447.

84. Pour consoler Paola Foresi de ses soucis, Colombini lui envoie des chanteurs de laudes gésuates, Barna di Montalcino et Boccia (G. Colombini, Le Lettere, Pref. P. Misciattelli, ouvr. cité, p. I8-20). Pour Misciattelli, la correspondance de Colombini anticipe celle de Catherine en témoignant de l'amitié créatrice qu'elle sait susciter. À ce sujet il rappelle qu'assistant à la mort d'un compagnon, le Gésuate Marco d'Arezzo le prie de l'appeler depuis l'Outre-Tombe, puis il tombe effectivement malade et explique à ses frères que ses tremblements ne sont pas une souffrance, mais de la joie de rejoindre l'Éternel. Ce sens de l'amitié fraternelle qui unit les frères par-delà la mort n'est pas sans rappeler certains récits des Fioretti, comme le chapitre 43 au sujet du frère Conrad d'Offida (Les Fioretti de saint François, dans S. François d'Assise, Documents..., ouvr. cité, p. II75-II77). 
Si ses nobles origines et sa dignité d'abbesse valaient à Paola Foresi le respect dû à son rang, Catherine ne pourra, quant à elle, se prévaloir d'aucun titre de noblesse ni d'aucune charge ecclésiastique, dans la mesure où cette jeune femme issue de la petite bourgeoisie artisane est restée tertiaire toute sa vie. Dans ce sens, sa légitimité la rapprocherait plutôt d'un autre personnage qui a marqué la conversion de Colombini : Marie l'Égyptienne, dont nous avons vu qu'il avait lu la légende sur le conseil de sa propre épouse. Il n'est pas certain que Catherine ait partagé cette lecture, car ce personnage n'est jamais cité en exemple dans ses lettres ${ }^{85}$. On ne peut toutefois pas s'empêcher de reconnaître, dans la leçon salutaire infligée par Dieu à l'orgueilleux ermite édifié par les paroles inspirées d'une simple prostituée, la logique qui légitime la prise de parole des humbles face aux puissants, des laïcs face aux clercs, et en particulier des femmes incultes face aux savants, des prophétesses face aux docteurs et théologiens.

Nous trouvons le même type d'exemplum sous la plume du frère franciscain Arnaud, le confesseur et secrétaire d'Angèle de Foligno, quand il compare la visionnaire à la prophétesse Houlda qui reçoit le charisme de la prophétie pour humilier les savants coupables de transgresser la loi de $\mathrm{Dieu}^{86}$. C'est encore le même argument que met en avant Raymond de Capoue dans sa Légende Majeure pour expliquer que si Dieu a mis tant de sagesse dans la bouche d'une jeune fille, c'est probablement pour

confondre l'orgueil des hommes, de ceux surtout, qui, pleins de l'estime d'eux-mêmes, ne rougissent pas de se dire savants, alors qu'ils ne savent rien, sages alors qu'ils n'ont en rien goûté aux douceurs de la Sagesse divine. [...] Ce sont ces hommes, si je ne me trompe, que l'éternelle sagesse semble avoir résolu de confondre, par l'humble doctrine et les œuvres de merveilleuses de saintes jeunes filles... ${ }^{87}$

85. Cet exemple ne se trouve pas même cité dans sa lettre T276 à une prostituée de Pérouse où on aurait pu éventuellement attendre cette référence, et dans laquelle Catherine évoque en revanche le modèle plus connu de Marie-Madeleine.

86. A. de Foligno, Le livre des visions..., ouvr. cité, p. 28 : «Il n'est pas dans l'ordre ordinaire de la Providence qu'une femme enseigne et confonde la grossièreté des savants. Mais saint Jérôme, parlant de la prophétesse Olda, vers qui se faisait le concours des peuples, dit que, pour confondre la fierté de l'homme et la science prévaricatrice, le Seigneur a transporté sur la tête d'une femme le don de prophétie.» L'exemple de la prophétesse Houlda ou Hulda (II Rois, 22, I4) avait déjà été allégué par Jérôme et repris dans ce sens par saint Thomas (Summa theologica, dans Opera omnia, t. 2, Paris, Vivès, I882, IIa, Ttae, 5, Q. I7I-I78, et en particulier I7I et I75).

87. Raymond de Capoue, Vie..., ouvr. cité, p. 420-42I. Voir aussi l'appel divin entendu par Catherine, p. I29-I30: «En ce temps-ci, il y a un tel débordement d'orgueil, surtout parmi ceux qui se croient lettrés et sages, que ma justice ne peut attendre plus longtemps pour les confondre par un juste jugement. Mais parce que ma miséricorde règne toujours sur toutes mes œuvres, je vais commencer par infliger à ces orgueilleux une confusion qui leur sera salutaire et utile, s'ils veulent s'humilier en rentrant en eux-mêmes. Ainsi ai-je fait pour les Juifs et les Gentils, quand je leur ai envoyé des simples d'esprit, remplis par moi de la divine Sagesse. Je vais donc, pour confondre leur témérité, leur susciter des femmes ignorantes et faibles par nature, mais que j'aurai dotées d'une sagesse et d'une puissance divine." 
D'une certaine manière, Marie l'Égyptienne incarne déjà la légitimité de la parole prétendument simple des laïcs et exprimée en langue vernaculaire, face à l'arsenal théologique déployé par les clercs en latin, une légitimité dont Colombini se prévaut, dans le sillage de l'auteur du Cantique des créatures $^{88}$.

Sur le plan culturel, la veine populaire qui caractérise les lettres en siennois du Gésuate est, comme chez Catherine, aux antipodes de la savante exégèse en latin de type joachimite à laquelle Catherine se montre par ailleurs très hostile ${ }^{89}$. Colombini ne semble en effet pas particulièrement instruit et la culture marchande dans laquelle il s'est formé laisse penser qu'il est vraisemblablement plus aguerri à faire les comptes qu'à lire et écrire le latin. L'importante correspondance en langue vulgaire qu'il entretient suite à son bannissement, avec sa fille et les sœurs du monastère de Santa Bonda, témoigne plutôt d'un style efficace et concentré, ne se perdant point en détours inutiles et qui est vraisemblablement aussi celui de ses prédications.

On retrouvera sous la plume de Catherine ce style du sermo humilis, d'empreinte là aussi typiquement franciscaine. Inauguré par les Pères de l'Église sur le modèle même de la Bible, le sermo humilis est un style simple permettant d'exprimer des idées spirituelles élevées. S'opposant aux intellectuels païens qui en critiquaient la rudesse, Augustin le défendit, dans ses Confessions, au nom de l'universalité du public auquel est destiné le message chrétien ${ }^{\circ \circ}$. Progressivement supplanté, au fil du Moyen Âge par un style exégétique complexe, le sermo humilis trouve un représentant tardif en saint François qui, par son charisme personnel, lui rend son efficacité originelle en l'appliquant à la langue vulgaire ${ }^{91}$. C'est bien dans cette veine

88. Dans le cadre d'une réflexion plus vaste sur la question de la légitimité de la parole du laïc dans le domaine de la vie religieuse a priori réservé aux clercs, on pourrait penser aussi à l'exemple d'Oza ou Uzza (2 Ch. 13, 7-II) brandi par Dante dans son épître latine aux cardinaux italiens, réunis en conclave à Carpentras en I3I4 (Dante, Euvres complètes, trad. et com. André Pézard, Paris, Gallimard, 1965, p. 777-788, et en particulier p. 78I).

89. Voir la lettre $\mathrm{T}_{343}$ dans laquelle Catherine condamne les spéculations savantes sur l'Apocalypse: «Molti sono che, senza umilità e senza studio in cognoscere i defetti loro, assottiglieranno lo intelletto, e con l'occhio tenebroso vorranno vedere la profondità de la santa scrittura, e vorrannola 'sponere e intendere a loro modo: studieranno l'Apocalipse non con umilità né col lume de la fede - ma con infedelità s'avviluppano in quello di che non sanno riuscire; e cosi trae de la vita la morte, e del lume tenebre. La mente che debba essere piena di Dio, ed ella è piena di fantasie; e 'l frutto che ne li seguita è la confusione e la tenebre de la mente. Questo gli adiviene perché inanzi che elli scendesse volse salire."

90. Andrea Battistini et Ezio Raimondi, Le figure della retorica, una storia letteraria italiana, Turin, Einaudi, I984 (I), I990, p. I6.

91. Ibid. p. 24. Au sujet de la manière de prêcher de saint François, voir Tommaso da Celano, Vita Secunda, dans S. François d'Assise, Documents..., ouvr. cité, ch. 30, 86, p. 266, et en particulier sur sa faculté d'adapter son sermon à son auditoire (ibid., ch. 73, I07, p. 4I4-4I5), sur son prêche devant les cardinaux (ibid., ch. 27, 73, p. 254), sur son jugement nuancé sur les prédicateurs (ibid., ch. I22, I63, p. 46I-462 et ch. I23, I64, p. 462). 
que s'inscrit Catherine et avant elle Colombini, dont l'efficacité expressive est reconnue par Messer Domenico lui-même, savant théologien qui avoue n'avoir jamais rien lu d'aussi édifiant sur l'amour unitif dans toute la littérature religieuse que la lettre que Colombini lui a adressée à ce sujet $^{92}$. Et ce n'est sans doute pas un hasard si pour Raymond de Capoue, la prose de Catherine apparaît comme un calque en langue vulgaire de la prose latine d'Aurelius Augustinus ${ }^{93}$.

La critique a longuement débattu sur l'identification de l'Arbor Vitae d'Hubertin de Casale comme source littéraire de Catherine de Sienne ${ }^{94}$. En vérité, cette exégèse érudite s'inscrit dans une culture savante dont Catherine condamne les excès de spéculation, comme nous l'avons vu. Comme le rappelle André Vauchez, les saintes et les bienheureuses du $\mathrm{XIV}^{\mathrm{e}}$ siècle sont souvent considérées comme «les véritables continuatrices et héritières du Pauvre d'Assise et de son message, que les hommes auraient trahi en voulant le rendre intellectuel et rationnel ${ }^{95}$ ". Les dérives à la fois savantes et paupéristes des deux branches de l'ordre, avaient déjà été dénoncées dès le début du siècle par Dante ${ }^{96}$. C'est en revanche chez ces saintes et bienheureuses du Trecento que l'on retrouve l'écho le plus fidèle de cette intériorisation de la spiritualité inaugurée par la stigmatisation de saint François. Et en effet, selon Catherine, qui aurait elle-même reçu des stigmates intérieurs, la leçon du rédempteur n'est pas à rechercher dans les livres, mais à lire sur le corps même du Christ, véritable parchemin en peau d'Agneau, dont les plaies sanglantes sont les initiales enluminées des rubriques :

Sur le rapport de François avec les Saintes Écritures, sa manière de les assimiler (T. da Celano, Vita Secunda, ouvr. cité, ch. 68, IO2, p. 4IO-4II) et la simplicité avec lesquelles il convient de les approcher (ibid., ch. I42, I89, p. 482, où l'on trouve l'image de l'écorce et de la mœlle reprise ensuite par Catherine. Sur le rapport de saint François avec la Bible en général, voir les pages d'A. Vauchez, François d'Assise, ouvr. cité, p. 38I-393.

92. Nous trouvons en effet dans cette lettre, sous la plume d'un théologien lettré, une proclamation d'humilité face à l'inspiration mystique de Colombini : "ho letto tutto el Vecchio e Nuovo testamento, Vite e Collazioni dei santi Padri, quasi tutti gli scritti di Deonisio, el ccompendio della Sacra Teologiala Deosoebia, l'arilogio della Sapienza, il testo della mistioca Teologia e altri molti libri teologici, e mai non compresi in un tanto lume di verità dell'amore unitivo, quanto l'ho compreso per la vostra lettera, e sono si forte invilito che mi pare essere un animale bruto, considerata la mia miseria e la mia ignoranza" (G. Colombini, Le Lettere, Prefazione di P. Misciattelli, ouvr. cité, p. 22).

93. Voir Raymond de Capoue, Vie..., ouvr. cité, p. 424.

94. L'Arbor vitae avait été désigné comme la source principale de Catherine par Alvaro Grion (Santa Caterina da Siena, Dottrina e fonti, Brescia, 1953) à qui Giacinto d'Urso opposa les nombreuses sources thomistes de la tertiaire dominicaine ("Il pensiero di Caterina da Siena e le sue fonti", La Sapienza, n 3 3-4-5, mai-octobre I954, anno VII, p. 335-388; "La cultura e i maestri di Santa Caterina», Santa Caterina, rivista bimestrale di Studi Cateriniani, Nuova Serie, anno V, fasc. 5, 1954, p. 22-32).

95. A. Vauchez, François d'Assise, ouvr. cité, p. 448.

96. Voir les fameux vers I24-I26 du Paradis où Bonaventure dénonce les dérives des deux branches de l'ordre franciscain. 
E[Dio] àcci dato el libro scritto, cioè el Verbo dolce del Figliuolo di Dio, il quale fu scritto in sul legno de la croce, non con incostro, ma con sangue, co' capoversi delle dolcissime e sacratissime piaghe di Cristo. E quale sarà quello idiota grosso, e di sì basso intendimento che non le sappi lègiare? Non ne so neuno, se no gli amatori propi di loro medesimi, e questo l'adiviene non perché non sappino, ma perché non vogliono. Sì ch'egli è scritto, unde noi troviamo nel capoverso dè piei che gli à confitti acciò che confichiamo l'affetto in lui, spogliandolo d'ogni disordinata volontà, che non cerchi né voglia altro che Cristo crocifisso; volendo giognare al Padre eterno col mezzo di questa Parola incarnata, libro scritto (T309)97.

C'est en particulier en raison de cette exemplarité immédiatement accessible du Christ, brandie comme alternative à la culture savante des clercs, que Catherine s'inscrit dans le sillage de Colombini et plus globalement dans l'héritage de saint François.

La mise en perspective des figures de Giovanni Colombini et Catherine de Sienne appellerait, on le voit, de plus amples développements, en particulier une analyse stylistique comparée de leurs correspondances respectives, qui ferait apparaittre les similitudes et les dissemblances à la fois doctrinales et rhétoriques entre les deux épistoliers. À ce premier stade de l'enquête, on ne peut que constater, au-delà d'une distinction très marquée sur la question de la pauvreté, des affinités de positions entre ces deux figures centrales et contemporaines de la vie spirituelle siennoise de la deuxième moitié du XIV siècle.

Tout d'abord, Colombini a pu incarner pour Catherine une figure vivante du franciscanisme des origines à la fois dans le rapport entretenu entre le laïc et la hiérarchie ecclésiastique et dans la soumission absolue proclamée envers le pontife. Mais plutôt que de parler de Colombini comme un modèle de Catherine, il conviendrait peut-être mieux de qualifier Catherine de continuatrice de Colombini dans la mesure où elle reprend pour l'amplifier la dynamique restée in nuce chez le Gésuate, qu'il s'agisse de la portée de ses œuvres de pacification, de l'envergure de son apostolat ou de la teneur de son engagement au service de l'Église. Enfin, si Colombini a pu représenter pour Catherine une figure concrète du franciscanisme, c'est en particulier dans la légitimité qu'il accorde à

97. «Et [Dieu] nous a donné le livre écrit, c’est-à-dire le doux Verbe, Fils de Dieu, qui a été écrit sur le bois de la croix, non pas à l'encre, mais en lettres de sang, avec les initiales enluminées que sont les très douces et très saintes plaies du Christ. Et quel sera cet idiot mal dégrossi et de si vil entendement, qui ne sache les lire? Je n'en connais aucun, si ce n'est ceux qui sont épris d'eux-mêmes, et cela se produit, non pas parce qu'ils ne sauraient pas, mais parce qu'ils ne veulent pas. C'est pourquoi il est écrit, de sorte que nous comprenions, dans l'initiale enluminée de ses pieds cloués, que nous devons ancrer notre affection en lui, en dépouillant cette dernière de toute volonté désordonnée qui ne chercherait ni ne voudrait pas uniquement le Christ crucifié, si nous voulons atteindre le Père éternel par le moyen de la cette Parole incarnée, le livre écrit." 
la femme d'une part et d'autre part à la culture populaire, à la langue vulgaire et au sermo humilis. Il s'agit en fait là des deux versants d'une même démarche d'humilité d'empreinte très franciscaine et qui, selon la tradition chrétienne et la logique même de la kénose, consiste à s'abaisser volontairement à ce qui, dans la société, se situe aux antipodes du pouvoir et du savoir, et, dans le cas de Colombini, de la richesse ${ }^{98}$. On retrouvera chez Catherine ce renversement des valeurs mondaines, qui, dans le cas de Colombini, avait fait trembler le gouvernement des Douze. Si Raymond de Capoue fait silence sur Colombini dans la Légende Majeure, c'est peutêtre pour éviter d'attirer sur Catherine, dont il cherche à montrer qu' elle est en odeur de sainteté, l'odeur de soufre liée à cette rupture radicale que représentent les Gésuates soupçonnés d'affinités avec les Fraticelles et dont la réputation reste peut-être entachée malgré leur approbation par Urbain $V$ et ses successeurs.

Colombini semble donc avoir incarné à l'époque de Catherine un modèle vivant de franciscanisme, mais l'intense activité épistolaire qui rapproche les deux figures siennoises n'est en revanche pas une caractéristique franciscaine. Initialement engendrée chez Colombini par son statut d'exilé (en tant que tel, il n'est du reste pas un cas isolé99), l'écriture épistolaire répond ensuite chez lui aux nécessités d'une vie itinérante, non plus subie, mais choisie en réponse à une vocation missionnaire. Cet apostolat itinérant, qui n'était du reste pas étranger à saint François, renvoie au modèle des apôtres eux-mêmes et plus particulièrement à la prédication épistolaire de Paul, dont il serait également intéressant de comparer l'influence dans les lettres de Colombini et dans celles de Catherine.

98. A. Vauchez, François d'Assise, ouvr. cité, p. 446.

99. On peut penser par exemple au cas, bien que plus tardif, de la correspondance d'Alessandra Macinghi Strozzi étudié par Michelle Schuller («Le temps et l'espace dans les lettres d'Alessandra Macinghi Strozzi », Revue des études italiennes, t. $46, \mathrm{n}^{\mathrm{os}} 3-4,2000$ ). Comme le fera Catherine, Colombini souligne à maintes reprises dans ses lettres le rapport entre écriture et absence, en particulier dans les lettres aux moniales de santa Bonda : "Io avarei desiderio di scrivare a tutte voi, mo maggiormente d'essare con voi, non posso più; io ispero che Iddio quando che sia adempirà e nostri santi desideri" (G. Colombini, Le Lettere..., a cura di Dino Fantozzi, ouvr. cité, vol. II, p. I2I). 